\title{
Wissen handelbar gemacht? Politik und Patente
}

\author{
Irene Troy / Raymund Werle
}

MPIfG Journal Article

Leonhard Dobusch, Sigrid Quack: Organisation und strategisches Framing privater Regulierung: Urheberrecht zwischen Kreativität und Verwertung. In: Andreas Busch, Jeanette Hofmann (Eds.): Politik und die Regulierung von Information.

Politische Vierteljahresschrift, Sonderheft (46), 273-318 (2012). Nomos

The MPIfG Journal Articles series features articles by MPIfG researchers and visiting scholars published in peer-reviewed journals. Max Planck Institute for the Study of Societies (MPIfG) Cologne | www.mpifg.de

\section{Einleitung'}

Moderne Gesellschaften verstehen sich als Wissensgesellschaften. Zumindest teilen sie die Vision, sich auf dem Weg von der Industriegesellschaft zur Wissensgesellschaft zu befinden (Stehr 1994; 2002, S. 61-73). Selbstredend ist Wissen auch für die Industriegesellschaft von erheblicher Bedeutung, und es war von jeher eine Triebkraft wirtschaftlicher Entwicklung (Mokyr 2002; UNESCO 2005). Neu ist die hohe Geschwindigkeit, mit der ständig neues Wissen entsteht und Bestehendes entwertet wird. Gleichzeitig ändern sich auch die Bedingungen der Erzeugung und Nutzung von Wissen (David/ Foray 2002). Hinsichtlich seiner Nutzung erscheint Wissen als Produktionsfaktor, der gegenüber den herkömmlichen Produktionsfaktoren Land, Arbeit und Kapital stark an Bedeutung gewonnen hat (Drucker 1993; Willke 2007). Grundsätzlich hat Wissen als Produktionsfaktor den Charakter eines Wirtschaftsguts, das als solches zirkulieren kann (vgl. Coriat/ Weinstein 2008). Dies gilt speziell für technologisches Wissen.

Der Prozess der Transformation der Industriegesellschaften in Wissensgesellschaften ist überlagert von einer auf weit verbreiteten neoliberalen Überzeugungen beruhenden Politik, die den Marktmodus als dominante Governanceform der Gesellschaft verankern will (Djelic 2006). In dieser Diktion garantiert (nur) der Markt die effiziente Allokation und Nutzung von Ressourcen und damit letztlich auch von Wissen. Seit den frühen 1980er Jahren wurden zunächst in USA und den westlichen Industriestaaten politische Maßnahmen zur Vermarktlichung gesellschaftlicher Bereiche ergriffen, die bislang nicht oder nur sehr eingeschränkt der Marktlogik unterworfen waren. In einem ersten Schritt wurden die meist öffentlichen Versorgungsmonopole im Bereich der technischen Infrastruktur privatisiert und liberalisiert (Höpner et al. 2009). Hier zeigte sich bereits deutlich, dass Märkte keineswegs voraussetzungslos sind oder die quasi natürliche Form des wirtschaftlichen Austauschs darstellen. Vielmehr werden sie zumeist kollektiv organisiert, und häufig sind es - wie im Falle der Versorgungsmonopole - staatliche Institutionen, die die Gesetze und Regeln festlegen, welche einen Markttausch ermöglichen sollen. Hierauf verweist auch Neil Fligstein, der betont, dass man die Bedeutung des Staates für die modernen Märkte gar nicht überschätzen könne. Als ein Beispiel nennt er die vom Staat erteilten Patente, die den Firmen das rechtlich garantierte Monopol einräumen, mit dem geschützten Wissen Gewinne zu erzielen (Fligstein 2001, S. 3, 4, 45-53). Ähnlich wie Eigentumsrechte in anderen

1 Unser besonderer Dank für sehr konstruktive Kritik gilt den Herausgebern dieses Sonderhefts, zwei Gutachtern sowie Sebastian Haunss. Für wertvolle Forschungsassistenz danken wir Robin Kremer. 
Bereichen erscheinen Patente als eine wichtige das Funktionieren eines Marktes für Wissen und dessen Diffusion fördernde Institution. Speziell die industrieökonomische und die Managementliteratur betont genau diesen Aspekt und verweist auf (behauptete) einzelwirtschaftliche und gesamtwirtschaftliche Vorteile eines Marktes für Wissen. Sie konstatiert aber auch bestehende Unzulänglichkeiten bzw. Funktionsdefizite eines solchen Marktes (Arora et al. 2001; Lichtenthaler 2005).

In diesem Beitrag diskutieren wir zunächst einige grundsätzliche Probleme der Kommodifizierung von Wissen in Form von Patenten. Dann skizzieren wir im historischen Rückblick den politischen Prozess der Stärkung und Globalisierung des Schutzes geistigen Eigentums, um auch die politische Verankerung des gehandelten Gutes zu veranschaulichen. Wie dann die Analyse der aktuellen Entwicklung seit den 1980er Jahren zeigt, markiert diese einen „weltweiten Trend zur Privatisierung des Wissens" (Meier 2005, S. 492), wenn auch nicht unbedingt zu dessen Vermarktlichung, in einer Periode, in der auch die Privatisierung und Liberalisierung anderer Bereiche einsetzt. Wir konzentrieren uns dabei im Wesentlichen auf Patente, die neues technologisch nützliches Wissen schützen (sollen). Anschließend untersuchen wir mit Blick auf die mögliche Herausbildung eines marktmäßigen Handels mit Patenten, welche politischen und unternehmerischen Initiativen den Handel fördern (sollen) und ob sich ein Markt für Patente entwickelt hat. Die Patentpolitik - so unsere erste These - hat traditionell sehr viel stärker den Schutz geistigen Eigentums und der Investitionen in Innovationen als die marktmäßige Handelbarkeit patentierten Wissens angestrebt. Dies gilt tendenziell auch für die vergangenen stärker neoliberal geprägten Jahrzehnte. Hier finden wir relativ häufiger private unternehmerische Initiativen der Marktschaffung. Generell - so unsere zweite These - sind sowohl politische als auch private Maßnahmen der Förderung des Handels mit dem Problem konfrontiert, dass Wissen nur eingeschränkt kodifizierbar ist. Es ist unsicher, ob patentiertes Wissen ohne komplementäres nicht kodifiziertes Know-how wirtschaftlich genutzt werden kann. Patente haben sich daher nur sehr begrenzt als marktmäßig handelbare Wirtschaftsgüter etabliert. Wenn und soweit patentiertes Wissen übertragen wird, erfolgt dies überwiegend nicht als punktuelle Markttransaktion, sondern in Form von Kooperationsvereinbarungen zwischen Lizenzgeber und Lizenznehmer.

\section{Patentiertes Wissen als handelbares Wirtschaftsgut}

In seiner Analyse der sich herausbildenden Wissensgesellschaft argumentiert Peter Drucker, dass in ihr die Konkurrenz viel größer sein wird als in der Industriegesellschaft, ,for the simple reason that with knowledge being universally accessible, there are no excuses for nonperformance" (Drucker 2007, S. 233). Aus dieser Perspektive erscheint Wissen als unerschöpfliches öffentliches Gut, von dessen Gebrauch grundsätzlich niemand ausgeschlossen werden kann. Im Gegensatz zu anderen öffentlichen Gütern, den sogenannten "common pool resources“ (Ostrom 1990), ist eine Übernutzung der Wissensallmende (knowledge commons) ausgeschlossen. Eine reichhaltige Allmende fördert den wirtschaftlichen Wohlstand der Wissensgesellschaft. Ist neues nützliches Wissen jedermann zugänglich, 
so entfaltet es großen gesamtwirtschaftlichen Nutzen. Die Verbreitung des Wissens verursacht zumeist nur relativ geringe Kosten. Hingegen kann es sehr aufwändig sein neues Wissen zu schaffen (Foray 2004, S. 14-19). Wenn solches Wissen dann allgemein frei und kostenlos zugänglich ist und nicht privat angeeignet werden kann, werden daher ökonomisch rational kalkulierende Akteure wegen der Trittbrettfahrer-Problematik private Investitionen in die Produktion neuen Wissens in der Regel unterlassen. ${ }^{2}$ In der Terminologie der Ökonomen ist dies ein Fall von Marktversagen.

Hier kommt, worauf Fligstein (2001) verweist, der Staat ins Spiel. Staatlich gesetzte geistige Eigentumsrechte, auch gewerbliche Schutzrechte genannt, können sicherstellen oder zumindest dazu beitragen, dass die Investoren die ökonomischen Früchte ihrer Erfindungen ernten können. Wichtige Schutzrechte sind das Urheberrecht und das Patentrecht. Sie sind nationales Recht, können sich also von Land zu Land unterscheiden und haben sich auch historisch unterschiedlich entwickelt. In ihrer grundsätzlichen Ausgestaltung weisen sie jedoch inzwischen viele Ähnlichkeiten auf.

Das Urheberrecht regelt den Schutz persönlicher geistiger Schöpfungen, insbesondere Werke der Literatur, Musik, Wissenschaft und Kunst, aber auch Computerprogramme (Software), ohne dass das jeweilige Werk angemeldet oder der Rechtschutz beantragt werden muss. Der Urheber hat das alleinige Recht der Verwertung seines Werkes. Dieses Recht kann er an Dritte abtreten, mit denen er die Bedingungen der Verwertung vereinbart. Die Dauer des Urheberrechtsschutzes variiert international, ist aber im Allgemeinen sehr lang. In Deutschland wie in vielen anderen Ländern erlischt das Recht erst 70 Jahre nach dem Tode des Urhebers.

Anders als Urheberrechte werden Patente, auf die sich dieser Beitrag konzentriert, nur auf Antrag und nach einer Prüfung durch eine Patentbehörde erteilt und registriert. Patentiert werden können grundsätzlich nur Erfindungen und keine Entdeckungen, wobei deren Abgrenzung voneinander schwierig und politisch umstritten ist, wie etwa die Auseinandersetzung um die Patentierbarkeit von DNASequenzen gezeigt hat (Schneider 2003). ${ }^{3}$ Die Erfindungen müssen neu sein und auf einer aus der Sicht eines Experten nicht trivialen, aber nachvollziehbaren erfinderischen Tätigkeit beruhen. Zudem sollen sie gewerblich anwendbar (in USA "nützlich“) sein, also etwa die Wissensgrundlage für eine neue oder verbesserte Technik bilden. Patente schützen demnach neues nützliches technisches Wissen. Sie können einem individuellen Erfinder, aber auch einer Organisation, etwa einem Unternehmen oder einer Forschungseinrichtung, erteilt werden. Der Patentschutz beträgt regulär in den meisten Ländern 20 Jahre, vorausgesetzt das Patent

2 Diese Problematik darf allerdings auch nicht überschätzt werden. Wie aus der Innovationsforschung bekannt ist, erfordert die Absorption neuen Wissens oft ein hohes Maß an Expertise, Know-How und Flexibilität, über das nur wenige potentielle Trittbrettfahrer verfügen (vgl. Dolata 2009).

3 Für den Wirtschaftshistoriker Mokyr gehören Entdeckungen in die Kategorie des „propositional knowledge“, während Erfindungen „prescriptive knowledge“ verkörpern. Historisch, so der Autor, wurde propositionales Wissen, speziell wissenschaftliches Wissen, als öffentliches Gut behandelt, während präskriptives, technologisches Wissen von deren Erfindern entweder geheim gehalten oder nur patentrechtlich geschützt öffentlich zugänglich gemacht wurde (Mokyr 2002: S. 37). 
wird regelmäßig verlängert (Aufrechterhaltung), was wie auch schon die Anmeldung und Prüfung eines Patents mit nicht unerheblichen Kosten verbunden ist. Die Patentschrift, die spätestens mit der Erteilung veröffentlicht und somit frei zugänglich wird, beschreibt die Erfindung und formuliert Schutzansprüche, die alle möglichen, auch zukünftigen Implementierungen mit dem Ziel abdecken, dass die der Erfindung zugrunde liegende Idee geschützt wird.

Das Patent ist ein absolutes Ausschlussrecht. Zwar wird das patentierte Wissen veröffentlicht, doch dürfen es Dritte ohne Zustimmung des Rechtsinhabers nicht nutzen, zumindest nicht für gewerbliche Zwecke. Für seine Zustimmung kann er eine Zahlung verlangen. Erst nach Ablauf der Schutzfrist ist das patentierte Wissen für jeglichen, also auch den kommerziellen Gebrauch freigegeben. Patente verknappen das Wissen künstlich und schaffen zudem zeitlich begrenzte Alleinstellungen, die es erleichtern, mit einer Innovation einen wirtschaftlichen Gewinn zu erzielen (Landes/ Posner 2003, S. 8). Sie helfen so auch, das TrittbrettfahrerProblem zu reduzieren. Wissen bekommt den Charakter eines privaten Wirtschaftsgutes, über das nur dessen Eigentümer verfügen darf. Das Patent trägt also dazu bei, Märkte für Wissen zu konstituieren, indem es dieses verknappt und festlegt, wer rechtmäßiger Eigentümer ist und über die Verwertungsrechte verfügt. ${ }^{4}$ Tatsächlich gibt es Ähnlichkeiten, aber auch Unterschiede zwischen Patenten und materiellem physischen Eigentum, die vor allem von Juristen und Ökonomen mit Blick auf deren wirtschaftliche Konsequenzen diskutiert werden (Landes/ Posner 2003). In diesem Zusammenhang heben Bessen/ Meurer (2008, S. 10, 53) besonders hervor, dass Patente im Gegensatz zu materiellem Eigentum „fuzzy and unpredictable boundaries" haben, was eine Quelle von Unsicherheit bedeutet. Die verstärkt feststellbare Tendenz, gewerbliche Schutzrechte in enger Beziehung zum juristischen Eigentumskonzept zu sehen, und hiermit verbunden auch die Neigung, geistiges ähnlich wie physisches Eigentum zu behandeln, hat - zunächst speziell in USA - die Aufmerksamkeit der Gerichte auf die Trittbrettfahrer-Problematik gelenkt (Lemley 2005). Nur ein wirksamer rechtlicher Schutz würde es ermöglichen, Trittbrettfahren zu verhindern und das geistige Eigentum vollständig zu besitzen. Wenn es allerdings nur um die wirtschaftliche Aneignung von Wissen geht, das nicht weitergegeben werden soll, stellen Geheimhaltung, Verschlüsselung oder die Errichtung eines technischen Walls zum Schutze des Wissens wirksame Alternativen zum Patent dar (Harvey/ McMeekin 2007, S. 27-30, 177-183; vgl. auch Levin et al. 1987; Cohen et al. 2000; Blind et al. 2003).

Mit technischen Beschreibungen, Zeichnungen, Daten und auch Formeln enthält die Patentschrift kodifiziertes Wissen, das sich in dieser Form übertragen, speichern und reproduzieren lässt (Dasgupta/ David 1994, S. 493). Jedoch kann nicht alles Wissen, das im Kontext des Erfinders entstanden und verankert ist, problemlos kodifiziert werden (Von Hippel 1994; Nonaka/ Takeuchi 1995; Howells 1996; Zucker/ Darby/ Brewer 1998). Zudem sind einige Elemente als implizi-

4 Der Patentinhaber kann das patentierte Wissen Dritten natürlich auch kostenlos zur Verfügung stellen und diese gegebenenfalls vertraglich verpflichten, von ihnen entwickeltes komplementäres Wissen ebenfalls kostenlos zur Verfügung zu stellen. Diese Praxis ist in der Entwicklung von Software (Open Source) verbreitet (vgl. West 2006; Sebald 2008; Brand/Holtgrewe 2010). 
tes Wissen („tacit knowledge“) personengebunden und deshalb schwer übertragbar (Polanyi 1966). Allein auf der Basis "radikal dekontextualisierten“ (Weißbach 2010) und kodifizierten Wissens und ohne komplementäres nicht kodifiziertes Know-how sowie teilweise implizites Wissen lassen sich technische Innovationen selten erfolgreich transferieren und implementieren (Machlup 1984, S. 182-184; Dasgupta/ David 1994, S. 494; vgl. auch Kitch 1980). Dies stellt zweifellos ein Hindernis für den Handel patentierten Wissens dar, da häufig auch nicht kodifiziertes komplementäres Wissen übertragen werden muss, was zusätzliche Vereinbarungen über den Transfer solchen Wissens erfordert (Troy/ Werle 2008).

Einnahmen kann der Patentinhaber zum einen über den Verkauf von Produkten und Dienstleistungen erzielen, die auf der geschützten Innovation basieren. Zum anderen ist es aber grundsätzlich auch möglich, das innovative Wissen selbst $\mathrm{zu}$ handeln. Patentinhaber veräußern dabei entweder das Patent insgesamt, und die Erwerber treten in alle Rechte und Pflichten ein, oder die Parteien schließen einen Lizenzvertrag, mit dem bestimmte Nutzungsrechte übertragen werden. So kann der Patentinhaber die Nutzung des Wissens durch den Lizenznehmer regulieren (Boldrin/ Levine 2002). Die Transaktionen erfolgen gegen sofortige Bezahlung oder gegen Lizenzzahlungen über einen längeren Zeitraum. Hier handelt es sich jeweils um externe kommerzielle Patentverwertung, die prototypisch als Markthandel erfolgt. Dieser stellt insofern eine besondere Form dar, als er eine soziale Struktur des Austauschs von Rechten aufweist, welche, um eine minimale Konkurrenzsituation zu schaffen, durch potentiell mindestens drei Akteure konstituiert wird, die sich in der Rolle des Anbieters (Patentinhaber) und der Nachfrager für den Tausch entscheiden und ihr Handeln an Marktpreisen orientieren (Aspers 2009). Weniger marktliche Handelsformen, wie die Kreuzlizenzierung oder Patentpools, unterscheiden sich von der kommerziellen Veräußerung dahingehend, dass Patente zwischen Patentinhabern entweder direkt oder über einen Pool vermittelt getauscht werden. In beiden Fällen sichern sich Eigentümer wechselseitig Nutzungsrechte zu. Die Patentinhaber setzen dabei ihre Patente als bargaining chips ein, um Zugang zu komplementärem Wissen zu erlangen. Der Kreis der am Handel beteiligten Akteure ist relativ festgelegt und ergibt sich nicht aus den Marktkräften von Angebot und Nachfrage. Zudem werden die Patente in diesem Falle nicht gegen Geld getauscht. Als eine weitere weniger marktliche Handelsform ist die Lizenzierung von Patenten zwischen Mutter- und Tochtergesellschaften zu betrachten. Zwar können in diesem Fall Zahlungen erfolgen, doch die Transaktion ist nicht rein marktlich, sondern in der Terminologie Oliver Williamsons hierarchisch organisiert.

Aus der hier besonders interessierenden Perspektive der externen kommerziellen Verwertung von Patenten über Märkte unterscheidet sich Wissen in Bezug auf dessen Produktion und Handelbarkeit grundsätzlich nicht von anderen Wirtschaftsgütern. Dies entspricht der neoliberalen Sicht, für die, wie es der französische Philosoph Lyotard (1984, S. 4) ausdrückt, die Produktion von Wissen nicht mehr Zweck an sich sei, sondern mit dem Ziel erfolge es zu verkaufen. Die Käufer erwerben es zur Wertschöpfung im Rahmen der Produktion neuer Güter. Diesen Zustand der vollständigen Kommodifizierung erreicht Wissen wohl nur selten. Bob Jessop (2007, S. 117, 118) führt dies anknüpfend an Karl Polanyi (Polanyi 
1957, S. 68-76) darauf zurück, dass Wissen ähnlich wie Arbeit, Land und Geld bei Polanyi eine fiktive Ware sei, da es zwar marktmäßig handelbar gemacht, aber häufig nicht mit dem Blick auf einen Markttausch geschaffen werde. ${ }^{5}$ Die Herstellung und Verbreitung von Wissen basiere zumindest teilweise und insbesondere bei wissenschaftlichem Grundlagenwissen nicht auf Marktmechanismen, sondern sei durch nichtkommerzielle Anreize und Motive getrieben (vgl. hierzu Dasgupta/ David 1993; David 1998; Nelson 2004). Die kapitalistische Wissensgesellschaft erfordere jedoch die Kommodifizierung ihres konstitutiven Elements, des Wissens, das wie eine Ware behandelt werden müsse, damit über den Preis ein Ausgleich zwischen Angebot und Nachfrage erreicht werde. Der Warencharakter bleibe also fiktiv, was insbesondere Schwierigkeiten bei der Bestimmung des Tauschwertes von Wissen bereite (Jessop 2007, S. 121, 122). Die Fiktion wird hier noch verstärkt, wenn patentiertes Wissen als bargaining chip ähnliche Funktionen wie Geld ausübt.

Der Kodifizierung und Kommodifizierung und damit insbesondere der marktförmigen Handelbarkeit von Wissen sind also Grenzen gesetzt. In ihrer Analyse der Entwicklungen in den Biowissenschaften und des Rennens um die Entschlüsselung des menschlichen Genoms zeigen Mark Harvey und Andrew McMeekin (2007) paradigmatisch, wie sich hier, erstens, die Grenzen zwischen öffentlichem und privatem Eigentum an Wissen, marktmäßiger und nicht marktmäßiger Produktion und Diffusion von Wissen sowie Kooperation und Wettbewerb immer wieder verschieben und, zweitens, ökonomische und rechtliche Institutionalisierungen der Aneignung von Wissen dynamisch interagieren. Kodifizierbarkeit und Kommodizierbarkeit sind also keine historisch invarianten Eigenschaften von Wissen. Entsprechend variabel erscheint im historischen Verlauf auch die Politik der Zieldefinition und rechtlichen Gestaltung geistigen Eigentums, die nun mit Blick auf Patente betrachtet wird.

\section{Ursprung und Entwicklung von Patenten}

Die folgende skizzenhafte Darstellung analysiert aus einer politökonomischen Perspektive, welche in der Entwicklung des Patentsystems aufgekommenen rechtlich-politischen Motive und Ziele mit welchen Effekten für die Kommodifizierung und den Handel von Wissen relevant waren. Auf eine umfassende Beschreibung der engen Beziehung dieser Entwicklung zu der des Kapitalismus, zunächst in Europa und dann auch in anderen Regionen der Welt, wird weitgehend verzichtet (hierzu May 2000; May/ Sell 2006; Orsi/ Coriat 2006; May 2007).

\subsection{Herausbildung des modernen Patentsystems}

Erste Regeln der Patentierung (Privilegierung) von Erfindungen wurden bereits im 15. Jahrhundert in Venedig entwickelt und angewandt (vgl. May/ Sell 2006, S. 5874). Das Recht, technische Erfindungen kommerziell zu nutzen, galt lange Zeit als

$5,[\ldots]$ it is not produced for sale but is simply a gift of [human] nature or another 'aspect of man'“ (Jessop 2007:117). Vgl. aus einer anderen Perspektive auch Sebald (2008: S. 226-236). 
Privileg, das vom Adel oder Klerus verliehen wurde. Es ist mit modernen Patenten kaum zu vergleichen. ${ }^{6}$ Honoriert wurde die wirtschaftliche Wertschöpfung und weniger die individuelle Erfindung (vgl. Siegrist 2006). Das englische Statute of Monopolies von 1624, das sich noch ganz in dieser Tradition bewegte, erreichte dann schon einen beachtlichen Formalisierungsgrad (Machlup/ Penrose 1950, S. 2). Patente sollten dem ersten und wahren Erfinder erteilt werden, dem hiermit ein Verwertungsmonopol eingeräumt wurde. Erfindungen wurden patentiert, wenn sie neu waren, wobei das Neuigkeitserfordernis bis ins späte 18. Jahrhundert nur für das Territorium galt, für das dann auch das Patent erteilt wurde (MacLeod 1988; Khan/ Sokoloff 2001). Im Ergebnis diente daher der Patentschutz auch dazu, ausländische Technik mitsamt ihren Erfindern zu importieren. Ihnen wurde als Gegenleistung für die Unterrichtung einheimischer Handwerker in der neuen Technik Schutz vor Konkurrenz durch Monopolrechte an der Technik gewährt (David 1993). Angestrebt wurde hiermit, den Technologietransfer vom Ausland ins Inland zu fördern und so die einheimische Wirtschaft zu stärken.? Dieser Transfer erfolgte aber nicht in Form eines Patenthandels, sondern durch Anwerben der Personen, die über das gewünschte Wissen verfügten (Transfer von Humankapital). Die im Kapitel II aufgezeigten Probleme einer vollständigen Kodifizierbarkeit von Wissen stellten sich in diesem Falle eher nicht, da der Erfinder alles Wissen besaß.

Kennzeichnend für die erste Periode des Patentschutzes war auch, dass die institutionellen Rahmenbedingungen einen Handel von Patenten nur schwer zuließen. So war in Großbritannien der Kreis derjenigen klein, die Patente beantragten, da die Patentierungskosten sehr hoch waren und es außerdem für Erfinder außerhalb von London sehr schwer war, Informationen über bereits vergebene Patente zu erhalten. Zudem wurde der Handel dadurch erschwert, dass die Gültigkeit von Patenten unsicher war und Käufer von Patenten, die sich später als ungültig herausstellten, kaum auf Entschädigung hoffen konnten (Khan 2002). Wenn neben der Belohnung für Erfindungen durch Monopolrechte auch die Schaffung eines Marktes für Patente angestrebt war, so muss dies als in dieser Periode gescheitert betrachtet werden. Ausschlaggebend waren die rechtliche Unsicherheit bezüglich der Gültigkeit der Patente und die mangelnde Transparenz der Patentlandschaft.

Patentgesetze in ihrer modernen Form sind zunächst in USA entstanden. Viele europäische Länder zogen in der ersten Hälfte des 19. Jahrhunderts nach (Machlup/Penrose 1950, S. 1-3). Der Prozess begann mit dem amerikanischen Patentgesetz von 1793, das die weltweite Neuheit von Innovationen zur Patentierungsbedingung machte. Weitere moderne Kennzeichen waren niedrigere Anmeldegebühren, die sofortige Offenlegung von Patenten sowie deren gute

6 Privilegien konnten entzogen und neu verliehen werden, doch lag dies nicht in der Verfügungsmacht der Inhaber der Privilegien. Von einem Handel von Wissen unter Gleichen kann man deshalb nicht sprechen.

7 Mit Technologietransfer wird in der Literatur in der Regel die Übertragung von gewerblich nützlichem wissenschaftlich-technischem Wissen gemeint, wobei der Begriff der Technologie unpräzise bleibt (vgl. Arora et al. 2001: S. 3-6). Entscheidend ist, dass Wissen, auch in technischen Artefakten oder Personen ,eingebettetes “ Wissen, übertragen wird. 
Einsehbarkeit in allen Landesteilen (Khan 2002; Khan/ Sokoloff 2001). Ein zweiter wichtiger Schritt wurde mit dem Patentgesetz von 1836 getan. Es sah die Einrichtung eines Patentamts vor. Erfindungen wurden seitdem nicht mehr nur registriert, sondern direkt auf Neuheit und Nützlichkeit geprüft. Zudem erlaubte das Gesetz, dass auch Ausländer in USA patentierten (Dutfield 2003). Allerdings war der Schutz ihrer Erfindungen eingeschränkt, was in USA, das noch ein Netto-Technologieimporteur war, als legitimes Mittel verstanden wurde, wirtschaftliche Rückstände aufzuholen. Auch in anderen Ländern waren die Patentsysteme auf die spezifischen Interessen dieser Staaten oder einzelner Lobbygruppen zugeschnitten (May/ Sell 2006, S. 111-115; Khan 2002). Die konkrete Patentpolitik verfolgte vor allem industriepolitische und handelspolitische Ziele. Industriell entwickelte Länder belohnten und privilegierten Erfinder, während für industriell rückständige Länder wie damals die Niederlande und die Schweiz ein starkes Patentsystem, mit dem vor allem ausländische Erfindungen geschützt worden wären, industriepolitische Nachteile bewirkt hätte. Die Niederlande schafften deshalb den Patentschutz für einen Zeitraum von über 40 Jahren vollständig ab, und die Schweiz erließ erst gegen Ende des 19. Jahrhunderts ein Patentgesetz (Schiff 1971).

In den Niederlanden war der Widerstand gegen Patente allerdings auch von den Freihandelsideen liberaler Ökonomen geprägt. Machlup und Penrose (1950, S. 3) sprechen in diesem Zusammenhang von einem "Antipatent Movement“, das sich ab der Mitte des 19. Jahrhunderts in vielen Staaten formierte und besonders in den Ländern des Deutschen Zollvereins gegen Protektionismus und für Freihandel eintrat, sich aber letztlich nicht durchsetzen konnte. Von einer Abschaffung der Patente oder zumindest der hiermit verbundenen mehr als ein Jahrzehnt garantierten wirtschaftlichen Monopolstellung der Patentinhaber wären vermutlich Impulse für den Handel dieses Wissens ausgegangen.

Die in den modernen Patentgesetzen vorgesehenen Patentämter haben durch ihre Prüfverfahren die Rechtssicherheit von Patenten und damit auch den Schutz der Erfinder deutlich erhöht. Nach dem Erlass des Patentgesetzes von 1836, so argumentieren Lamoreaux und Sokoloff $(2001 ; 2007)$, führte dies in USA, wo auch die Anmeldegebühren relativ niedrig waren, zu einem starken Anstieg der Zahl der Patente und zu einer erheblichen Zunahme des Handels mit ihnen. Einzelne Patente wurden sogar mehrfach übertragen. In einer größeren statistischen Studie können die Autoren zeigen, dass sich gleichzeitig eine Gruppe von professionellen Erfindern herausbildete, die ihre Patente direkt weiter verkauften. ${ }^{8}$ Hier leistete eine zunehmende Zahl von über technische Expertise verfügenden Patentagenten und Anwälten Vermittlungsdienste, von denen auch potentielle Käufer profitierten. Viele dieser Intermediäre haben ihre Expertise erworben, als sie als Prüfer im Patentamt tätig waren. Sie konnten sich zudem als Patentagenten vom Patentamt registrieren lassen, was das Vertrauen in ihre Expertise stärkte. Ein wachsender Markt mit relativ vielen aktiven Intermediären erlaubte es immer mehr Erfindern, sich auf diese Tätigkeit zu spezialisieren, viele Patente anzumelden und diese zu veräußern. Es war ein Zusammenspiel von politischen Maßnah-

8 Zu ähnlichen Befunden gelangt Burhop (2009) für das Deutsche Reich zwischen 1877 und dem Ersten Weltkrieg. 
men zur Stärkung und Qualitätsverbesserung der Patente und von privaten Akteuren als Intermediäre oder spezialisierte Erfinder, das den marktmäßigen Handel von Patenten ermöglichte, weil es Transparenz, Vertrauen und Reputation entstehen ließ (Lamoreaux/ Sokoloff 2001; 2007). So entstand ein (nationaler) Markt für Patente, ohne dass er politisch explizit intendiert war.

Die unabhängigen Erfinder und mit ihnen die Patenttransaktionen verloren dann Ende des 19. Jahrhunderts wieder an Bedeutung, was nur teilweise auf eine Änderung der politisch-rechtlichen Rahmenbedingungen zurückzuführen ist. Eine gewisse Rolle spielte ein Urteil des US Supreme Courts aus dem Jahre 1871, das es Unternehmen erlaubte, die Angestellten zu verpflichten, ihr Patentrecht auf das Unternehmen zu übertragen. ${ }^{9}$ Bis dahin konnten nur die Erfinder selbst ein Patent erhalten (May/ Sell 2006, S. 118). Wichtig war wohl auch, dass die entstehenden Großunternehmen aus strategischen Gründen Forschung und Entwicklung in eigenen Abteilungen organisierten und internalisierten (vgl. Schumpeter 1996 [1943], S. 81-163; Nicholas 2003; Burhop 2009). Zudem wurden die Erfindungen offenbar auch komplexer und kapitalintensiver, was Einzelerfinder überforderte, aber auch eine vollständige Kodifizierung und damit einen einfachen Transfer des Wissens praktisch ausschloss (Khan/ Sokoloff 2001, S. 242, 243).

\subsection{Internationalisierung}

Mit der deutlichen Zunahme des internationalen Warenhandels in der zweiten Hälfte des 19. Jahrhunderts wuchs das Interesse speziell der exportstarken Staaten, die unterschiedlichen nationalen Patentsysteme stärker zu vereinheitlichen, um Patente auch im Ausland effektiv sichern zu können. Viele amerikanische Konzerne, die auf die internationalen Märkte drängten, begründeten ihren nationalen Erfolg auf Patenten. Diese genossen seit dem Ende des 19. Jahrhunderts aufgrund einer äußerst patentfreundlichen Rechtsprechung in USA einen weitgehenden Schutz und sicherten so die Monopolstellung dieser Konzerne (Sell 2003, S. 64-67). Daher kann es nicht überraschen, dass USA, um eigene Handelsvorteile zu sichern, auf eine Patentharmonisierung drängte, die möglichst stark dem eigenen Modell entsprach und sehr viel mehr dem Schutz von Erfindungen durch starke Patente (Investitionsschutz) als dem Handel derselben diente. Ein Resultat dieser Bestrebungen war das internationale Übereinkommen von Paris, die „Paris Convention for the Protection of Industrial Property“ im Jahre 1883 (Pariser Verbandsübereinkunft). Der Übereinkunft waren langjährige und bis heute aktuelle kontroverse Diskussionen vorausgegangen, die sich neben technisch-administrativen Punkten auf die Beziehung zwischen Patenten, Protektionismus, Freihandel und wirtschaftlicher Entwicklung bezogen (vgl. Jaffe/ Lerner 2004, S. 78-95). Sie mündeten in einen Kompromiss, der viele wichtige Punkte ausklammerte (Khan 2002; Dutfield 2003; May/ Sell 2006). Insbesondere USA und Deutschland als Verfechter eines starken Patentschutzes waren unzufrieden. Sie traten deshalb erst 1887 bzw. 1903 der auf der Basis der Übereinkunft gegründeten „Union for the Protection of In-

9 Diese Möglichkeit wurde nicht zuletzt auf Betreiben von Siemens auch im deutschen Patentgesetz von 1877 eröffnet. 
dustrial Property“ (Paris Union) bei. Die Übereinkunft bildet bis heute eine zentrale Säule des internationalen Rechts geistigen Eigentums und wurde von bislang 173 Staaten ratifiziert. Seit 1883 wurde sie mehrfach überarbeitet (zuletzt 1967), und die Rechte von Patentinhabern wurden gestärkt. Immer blieb aber die nationale Autonomie der Vertragsstaaten unangetastet (Dutfield 2003, S. 57).

Aus der Paris Union, die sich 1893 mit einer im Bereich des Urheberrechts angesiedelten Schwesterorganisation zusammenschloss, ging 1967 die World Intellectual Property Organization (WIPO) hervor, die seit 1974 eine Unterorganisation der Vereinten Nationen ist. Die WIPO bemüht sich um weltweite Stärkung und Harmonisierung der nationalen Regeln des Schutzes geistigen Eigentums. Nachdem im Rahmen der WIPO bereits 1970 mit dem Vertrag über die internationale Zusammenarbeit auf dem Gebiet des Patentwesens (PCT) die Grundlage für ein international vereinheitlichtes Rechercheverfahren für Patentanmeldungen vereinbart werden konnte, sind weitergehende substanzielle Harmonisierungsbemühungen auf der Basis eines „Substantive Patent Law Treaty“ trotz langjähriger internationaler Verhandlungen (zuletzt seit 2001) ergebnislos geblieben, was dem internationalen Handel patentierten Wissens natürlich nicht förderlich sein konnte. Ausschlaggebend für die Harmonisierungsprobleme sind die wirtschaftliche Konkurrenz der ökonomisch und technisch führenden Staaten, die mit dem Wirtschaftszweig und der Größe variierenden Interessen der Unternehmen, der NordSüd Konflikt, die unterschiedlichen Rechtstraditionen, die Eigeninteressen der nationalen Patentbehörden und der mit ihnen verbundenen Patentagenten und Anwälte und schließlich auch die Verhandlungs- und Entscheidungsregeln der WIPO (Eimer 2007; 2008).

Einige der genannten Faktoren haben auch dazu beigetragen, dass Bemühungen erfolglos blieben, ein einheitliches europäisches Patent oder zumindest innerhalb der Europäischen Union ein Gemeinschaftspatent zu schaffen. ${ }^{10}$ Immerhin gelang es, auf der Basis des 1973 unterzeichneten Europäischen Patentübereinkommens im Oktober 1977 mit der Europäischen Patentorganisation eine zwischenstaatliche Einrichtung mit inzwischen 37 Vertragsstaaten zu gründen, die Trägerin des Europäischen Patentamts ist. Die von dieser Behörde erteilten Patente haben in den Mitgliedstaaten zwar keine unmittelbare Geltung, das von ihr praktizierte Anmeldeverfahren und auch ihre Sachprüfungen werden aber von den nationalen Ämtern anerkannt, die auf dieser Grundlage dann ohne erneute Sachprüfung Patente erteilen.

Vor dem Hintergrund der Schwierigkeiten, eine internationale Angleichung oder gar Vereinheitlichung der nationalen Patentsysteme zu erreichen, erscheint das TRIPS-Abkommen zu „handelsbezogenen Aspekten der Rechte an geistigem Eigentum" als ein beachtlicher Schritt in Richtung auf eine neoliberal ausgerichtete Globalisierung des Schutzes geistigen Eigentums (Brand et al. 2008, S. 137-159;

10 Im Bericht der „Kok-Kommission“ vom November 2004 zur Implementierung der Lissabon Strategie für Wachstum und Beschäftigung wird der Europäische Rat aufgefordert die Patentierung computerimplementierter Erfindungen zu ermöglichen und „natürlich auch“ das Gemeinschaftspatent zu beschließen (S. 26). Beides ist nicht erfolgt bzw. gescheitert. <http://ec.europa.eu/education/policies/2010/doc/kok_de.pdf> 
speziell für die Biotechnologie Barben 2007, S. 151-159). Das Abkommen wurde 1994 im Zuge der Gründung der Welthandelsorganisation (WTO) im Rahmen der GATT-Verhandlungen vereinbart und direkt von 111 Staaten unterzeichnet. Für alle neu in die WTO aufgenommenen Mitglieder ist TRIPS automatisch verbindlich. Anders als die WIPO verfügt die WTO außerdem mit ihrem „Dispute Settlement Body " über unmittelbare Sanktionsmöglichkeiten bei Vertragsverletzungen, was dem Patentschutz zusätzliches Gewicht verleiht (May 2000, S. 68-90). ${ }^{11}$

TRIPS zielt darauf, Mindeststandards zum Schutz aller Arten geistigen Eigentums international durchzusetzen und gleichzeitig zu verhindern, dass die Schutzrechte als Hemmnisse des Technologietransfers wirken, ohne aber substanzielles nationales Recht zu harmonisieren. Jedoch ergibt sich eine gewisse Harmonisierung dadurch, dass die allgemeinen WTO-Prinzipien wie Meistbegünstigung und Reziprozität automatisch auch für TRIPS gelten (May 2000, S. 69).

Eine Untersuchung des TRIPS-Prozesses hat gezeigt, dass große international agierende Konzerne, speziell Unternehmen der Informationstechnik und Software, der pharmazeutischen Industrie und der Unterhaltungsindustrie und die sie jeweils vertretenden Verbände die Verhandlungen und ihr Ergebnis erheblich beeinflusst haben (Sell 1999; 2003). ${ }^{12}$ Es gelang den amerikanischen Konzernen, in der europäischen und südostasiatischen Industrie Verbündete zu gewinnen und mit ihrer Hilfe den jeweiligen Regierungen die handelspolitische Bedeutung des geistigen Eigentums $z u$ verdeutlichen. Für internationale Verhandlungen über den Schutz geistigen Eigentums im Rahmen eines "trade-based approach to intellectual property “ bildete GATT das zuständige Forum (Sell 2003). Hier drängten vor allem USA, später aber auch andere Industriestaaten mit hohem Exportvolumen, wie Deutschland oder Japan, auf eine Stärkung und Vereinheitlichung des weltweiten Schutzes geistigen Eigentums (vgl. Kahin 2003, S. 17). Aus handelspolitischer Sicht versprechen sich diese Länder Vorteile davon, dass die sich aus den jeweiligen Patenten ergebende Monopolstellung der Konzerne international gesichert ist und internationale "Produktpiraterie" verhindert wird. In jedem Fall gewinnen also diese Konzerne. Sie waren es schließlich auch, die darauf drängten, dass ihre Investitionen langfristig geschützt werden, um so ihre Profitchancen zu sichern (Braithwaite/ Drahos 2000, S. 65-75; Perez Pugatch 2004, S. 128-155; Drahos 2005, S. 146-148).

Statistiken und Berechnungen der Weltbank zeigen, dass unter den Staaten bislang im Wesentlichen nur USA von einem international verlässlichen Schutz geistigen Eigentums profitiert, weshalb es sich auch besonders stark dafür einsetzt. Wie Schaubild 1 erkennen lässt, weist USA für den Zeitraum ab 1970 in seiner Zahlungsbilanz regelmäßig einen Überschuss der Einnahmen über die Ausgaben

11 Die WIPO ist jedoch keineswegs bedeutungslos (May/ Sell 2006: S. 209-214). Ihr Einfluss liegt aber stärker im Bereich des Urheberrechtsschutzes, was insbesondere die Entstehung der sogenannten Internetverträge von 1996 und ihre Umsetzung in nationales Recht zeigt (vgl. Werle 2005).

12 Ähnliches gilt für den Einfluss der großen Medienkonzerne auf das amerikanische und internationale Urheberrecht (WIPO Internetverträge; US Digital Millenium Copyright Act) seit der Verabschiedung des US Copyright Act von 1976 (Landes/ Posner 2003: S. 403-413; vgl. auch Dolata 2008). 
aus internationalen Lizenzzahlungen auf. ${ }^{13}$ Dieser hat sich seit Mitte der 1980er Jahre, also lange vor dem TRIPS-Abkommen, rasch vergrößert und 2007 rund 43 Mrd. US\$ erreicht. Japan erzielte 2003 erstmalig einen Überschuss, der eine klar steigende Tendenz aufweist (2007 gut $6 \mathrm{Mrd}$. US\$). Deutschland hingegen weist durchgehend ein Defizit auf, das in den letzten Jahren zwar deutlich geschrumpft ist, seit 2004 jedoch wieder wächst (2007 fast $3 \mathrm{Mrd}$. US\$). Noch ungünstiger sieht die Bilanz zusammengenommen für die Mitglieder der europäischen Währungsunion aus. Hier ist das Defizit fast kontinuierlich gewachsen und hat 2007 rund 24 Mrd. US\$ erreicht. ${ }^{14}$ Modellrechnungen der Entwicklung des Wertes einheimischer und ausländischer Patente nach Abschluss des TRIPS-Abkommens sehen für einige Länder etwas günstiger aus. Doch zeigen auch sie, dass von dem aus der Harmonisierung resultierenden Einkommenstransfer mittelfristig nur wenige, nach diesen Berechnungen sechs Länder, profitieren: USA, Deutschland, Frankreich, Italien, Schweden und die Schweiz (McCalman 2001, S. 178). ${ }^{15}$

Für die Entwicklungsländer ist die mit dem TRIPS-Abkommen herbeigeführte Situation nachteilig. Sie sind in aller Regel Technologie-Importeure, und strenge Patentregeln bedeuten für sie Kostenbelastungen, sofern überhaupt patentiertes Wissen zum Erwerb angeboten wird (May/ Sell 2006, S. 185-189). Letztlich stimmten die Entwicklungsländer dem für sie unvorteilhaften Abkommen nur zu, weil sie befürchten mussten, bei bilateralen Handelsabkommen mit entwickelten Ländern, vor allem mit USA, noch stärker benachteiligt zu werden (Dutfield 2003; Meier 2005, S. 504-506). Der im TRIPS-Abkommen geforderte Technologietransfer, speziell auch in Form von ausländischen Direktinvestitionen in den Entwicklungsländern, und das Verbot des Missbrauchs geistigen Eigentums durch die Rechteinhaber zu Lasten des Transfers (Teil I: Artikel 7 und 8) haben sich als wenig wirksam erwiesen. Der Handel patentierten Wissens erhielt kaum Impulse. Im Gegensatz zu früheren industriellen Nachzüglern können die heutigen Entwicklungsländer allerdings nicht mehr durch einen schwachen Schutz geistigen Eigentums technologisch und wirtschaftlich aufholen. Somit erweist sich die vor allem von den exportstarken Ländern vorangetriebene Globalisierung des Patentschutzes in ihren Effekten eher als neomerkantilistisch denn als neoliberal (Landes/ Posner 2003, S. 410). Sie stärkt die Monopolposition der technologisch führenden Unternehmen in diesen Ländern und damit auch deren wirtschaftliche Macht, nicht jedoch den Handel patentierten Wissens.

13 Diese Zahlungen umfassen nicht nur Lizenzgebühren für Patente, sondern auch für Urheberrechte, Handelsmarken oder industrielle Prozesse („Royalties, License Fees, and Other Receipts and Payments for Intangible Rights Between Domestic and Foreign Persons").

14 In der Tendenz weisen Daten der OECD für den Zeitraum von 1970 bis 2001 in dieselbe Richtung (vgl. Perez Pugatch 2004: S. 52-56).

$15 \mathrm{Zu}$ den Einnahmen aus der Übertragung von Nutzungsrechten müssen, will man den gesamten wirtschaftlichen Vorteil eines Landes aus der Harmonisierung beurteilen, zumindest Teile der Auslandserlöse aus dem Verkauf patentgeschützter Güter und Dienstleistungen addiert werden. 
Schaubild 1: Lizenzeinnahmen und Lizenzausgaben für Patente, Urheberrechte und ähnliche Schutzrechte für ausgewählte Länder seit 1970 (Zahlungsbilanzwerte, in Mrd. US\$)
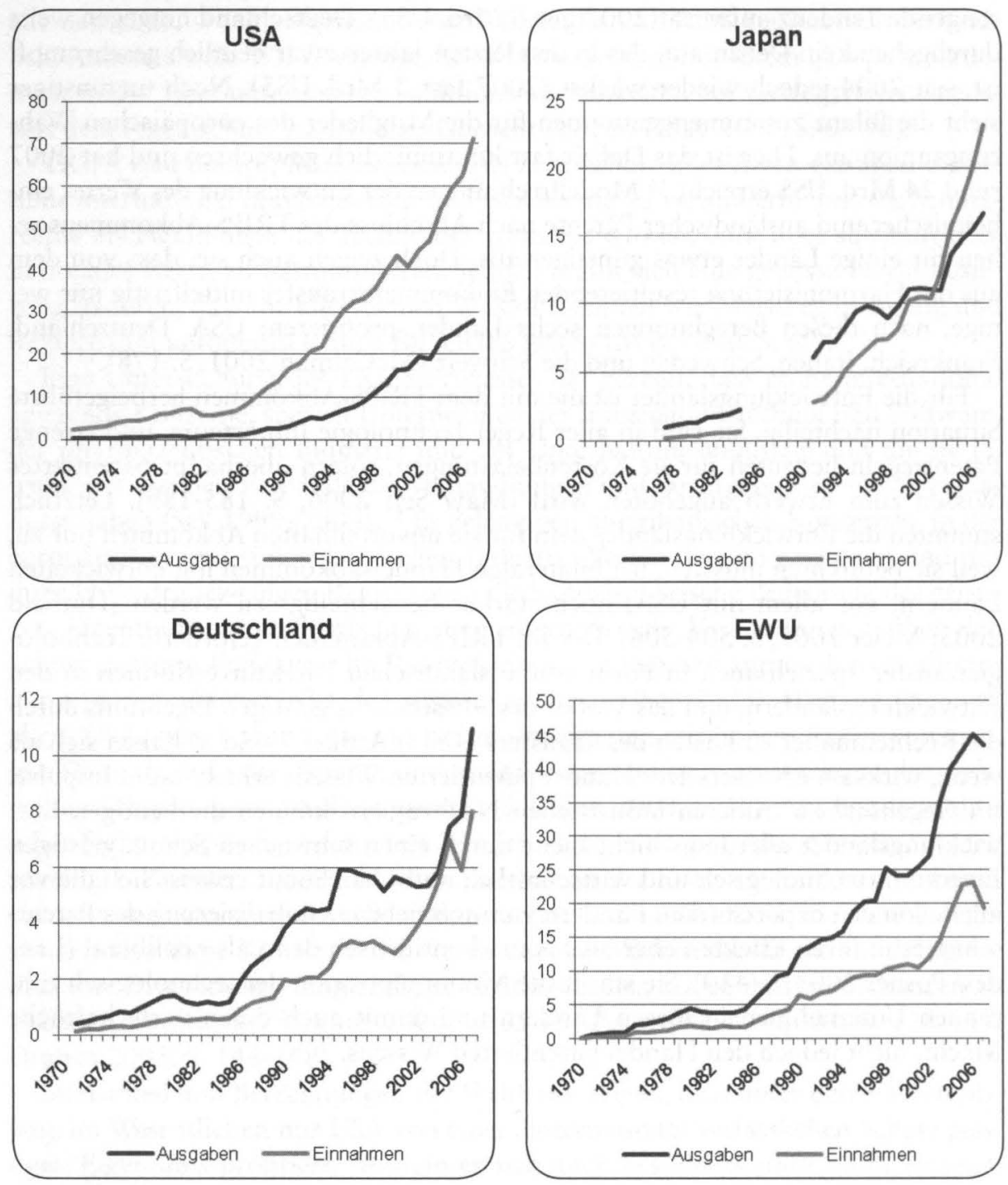

Quelle: Weltbank, World Development Indicators 2008 〈http://search.worldbank.org/all?qterm=World\%2odevelopment > 


\subsection{Aktuelle Entwicklung}

Der kurze Blick auf die Herausbildung des Patentsystems und seine internationale Ausbreitung hat gezeigt, dass unterschiedliche Motive und Ziele zusammenwirkten. Vorstellungen, dass der Erfinder ein quasi natürliches Recht habe, seine Erfindung als Eigentum zu besitzen oder für sie besonders belohnt und privilegiert werden müsse, wurden überlagert von industriepolitischen und handelspolitischen Interessen, die darauf zielten Patente einzusetzen, um neue Techniken vom Ausland ins Inland zu transferieren oder den heimischen Markt abzuschotten, wobei in bestimmten Fällen ein schwacher oder vollständig fehlender Patentschutz durchaus die bevorzugte strategische Variante darstellte. Das Patentrecht wie die immateriellen Eigentumsrechte insgesamt entwickelte sich also keineswegs geradlinig. „Die Entstehung der Schutzrechte war weder zwangsläufig noch folgte sie einer inneren, sich pfadabhängig fortschreibenden Logik von Sachzwängen “ (Hofmann/ Katzenbach 2006, S. 11). Vielmehr war das Patentrecht in der Regel auf den technologischen und wirtschaftlichen Entwicklungsstand der jeweiligen Volkswirtschaften, aber auch zunehmend auf die Interessen großer multinationaler Konzerne zugeschnitten. Besonders die schwergängigen Bemühungen, ein harmonisiertes internationales Patentregime durchzusetzen, sind Ausdruck und Folge historisch gewachsener Unterschiede zwischen den nationalen Patentsystemen und den global stark divergierenden Strategien der Staaten (vgl. Lerner 2002). Sie deuten zudem an, dass die Förderung des Handels mit patentiertem Wissen als solchem niemals hohe Priorität hatte.

Nicht nur international, sondern auch innerhalb der Staaten gab es keine geradlinigen Entwicklungen, was den Patentschutz betrifft. So bildeten sich in einer sehr patentfreundlichen Periode um die Wende zum 20. Jahrhundert in USA mächtige wirtschaftliche Monopole heraus, bis nach 1914 im Gefolge des Clayton Antitrust Act das den Patenten inhärente Potential zur Monopolbildung als Grund angeführt wurde, den wirtschaftlichen Nutzen von Patenten zu bezweifeln und sie oftmals für ungültig zu erklären (Eckl 2004, S. 74-81).16 Im nationalsozialistischen Deutschland hingegen leistete die Patentgesetzgebung, vor allem das Reichspatentgesetz von 1936, der politisch gewünschten Bildung von Kartellen und korporatistischen Wirtschaftsarrangements Vorschub und wirkte noch lange in die Bundesrepublik hinein (Seckelmann 2006).

Nach uneinheitlichen Entwicklungen in der Nachkriegszeit begann dann zunächst USA, den Schutz geistigen Eigentums zu erweitern und zu verstärken. Dies erfolgte auch in Reaktion auf eine Wirtschaftskrise in den späten 1970er und den frühen 1980er Jahren, als sich die Vorstellung verbreitete, das Ausland profitiere von unzureichend geschützten amerikanischen Erfindungen, aus denen es, speziell in den wissensintensiven High-Tech Bereichen, dann marktfähige Produkte machte (May/ Sell 2006, S. 141-153; Lemley 2005). Wichtige Impulse gingen von Ent-

16 Der bereits 1891 verabschiedete Sherman Antitrust Act war praktisch wirkungslos geblieben (Fourcade 2009: S. 36, 37). 
scheidungen des US Supreme Court aus. ${ }^{17}$ So erklärte er 1980 die Patentierung von gentechnisch manipulierten Organismen für zulässig. Bis dahin orientierte sich das US Patentamt an der „product of nature doctrine“, die besagt, dass Lebensformen und ihre strukturellen und funktionalen Komponenten nicht patentiert werden können. Ein Jahr später fiel auch das Patentierungsverbot für Software, die bis dahin nur urheberrechtlich in ihrer jeweils konkreten Ausdrucksform geschützt werden konnte (Harison 2008, S. 65-74). Das Gericht entschied, dass computerprogrammbezogene Erfindungen wegen ihres technischen Charakters patentierbar seien. Inzwischen gelten alle Kalkulationen und Prozeduren mit den ihnen zugrunde liegenden Algorithmen und Formeln als patentierbar, wenn sie von einer Maschine ausgeführt werden und Resultate produzieren, die „useful, concrete and tangible" sind (CSTB 2000, S. 192-195). Auf dem Wege der Gesetzgebung wurde beginnend im Jahr 1980 der Kreis der Patentberechtigten um Universitäten und öffentliche Forschungseinrichtungen erweitert, die nun grundsätzlich auch mit öffentlichen Mitteln finanzierte Erfindungen patentieren können. Insgesamt war es ein Zusammenwirken von legislativen, administrativen und gerichtlichen Aktionen in USA (Scherer 2007, S. 45; Landes/ Posner 2003, S. 414419), das dort die Pro-Patent-Ära einläutete (Cohen 2005; Granstrand 2000, S. 27-41). Die Europäische Union, Japan und die meisten anderen entwickelten Industriestaaten übernahmen - teils auf Druck von USA teils aus eigener Überzeugung - das amerikanische Muster im Grundsatz. Sie erweiterten die Patentierungsmöglichkeiten und den Kreis der Patentberechtigten (vgl. Park 2008).

So kann es nicht überraschen, dass seit spätestens Mitte der 1990er Jahre, in USA bereits zehn Jahre früher, die Zahl der Patentanmeldungen bei den Patentämtern drastisch zugenommen hat. Die „Patentexplosion“ (Hall 2005) lässt sich an den Statistiken der WIPO und der nationalen Patentämter ablesen. Die Zahl der Patentanmeldungen ist weltweit von knapp 1 Mio. 1995 auf knapp 1,8 Mio. im Jahre 2007 gestiegen, nachdem sie vorher viele Jahre relativ konstant war. Überproportional gewachsen ist sie in innovativen Technikfeldern wie der Informations- und Kommunikationstechnik und der Biotechnologie (European Patent Office 2007, S. 88-97). In USA hat das Wachstum, wie angedeutet, bereits früher eingesetzt. Von 1985 mit 117.000 Anmeldungen hat sich deren Zahl bis 1995 auf rund 212.000 fast verdoppelt, um bis 2008 noch einmal um mehr als $100 \%$ auf gut $456.000 \mathrm{zu}$ wachsen. ${ }^{18}$ In einigen Ländern, darunter Japan und Deutschland, stagniert die Zahl der Anmeldungen seit einigen Jahren, allerdings auf hohem Niveau (Schaubild 2, S. 168)..$^{19}$

Es sind aber nicht nur rechtliche und andere institutionelle Veränderungen, die zu dem starken Anstieg der Patentanmeldungen geführt haben. Vielmehr spielen,

17 In einer Serie von Entscheidungen betonte das Gericht, dass „monopoly power was the purpose of the patent grant" (Jaffe 2000: S. 532). Hierdurch erzielte Monopolrenten sind demnach gewollt und rechtmäßig.

18 Der Anteil amerikanischer Anmelder an der Gesamtzahl der Anmeldungen beim US Patentamt bewegt sich zwischen $50 \%$ und $60 \%$.

19 Für Deutschland erklärt sich die Stagnation durch die zunehmend genutzte Möglichkeit, die Anmeldung für ein deutsches Patent beim Europäischen Patentamt einzureichen, welches dann auch die Prüfung vornimmt. 
wie im nächsten Kapitel ausführlicher dargestellt, die Strategien der Unternehmen, für die innovatives technisches Wissen die Grundlage neuer Produktionsprozesse, Produkte und Dienstleistungen bildet, eine wichtige Rolle. Die Ergebnisse von Forschung und Entwicklung werden als zu schützende Vermögensgegenstände betrachtet. ${ }^{20}$ Bereits die Anmeldung eines Patents sichert einen provisorischen Schutz ab dem Tag der Anmeldung (Prioritätsdatum). Viele Anmelder nehmen ihren Antrag später wieder zurück oder verzichten auf die Neuheitsprüfung durch das Patentamt, etwa weil ihnen nach einiger Zeit ein besonderer Schutz nicht mehr notwendig erscheint. Solche Strategien sind ein Grund dafür, dass mit 30\% bis $40 \%$ der Anmeldungen sehr viel weniger Patente erteilt werden als zunächst Anträge eingehen. Dies deutet aber auch darauf hin, dass die unternehmerische Patentierungspolitik zumindest nicht vordringlich auf eine externe Kommerzialisierung neuen technischen Wissens zielt, weshalb auch nicht vom Anstieg der Zahl der Patentanmeldungen und Patenterteilungen vorbehaltlos auf einen entsprechend wachsenden Patenthandel geschlossen werden kann.

Insgesamt ist der weltweite Prozess der Erweiterung von Patentierungsmöglichkeiten nicht abgeschlossen. Er geht aber in den verschiedenen Regionen der entwickelten Welt unterschiedlich weit. Verglichen mit USA und speziell Japan - hier sind Computer-Programme inzwischen fast uneingeschränkt patentierbar - gelten Deutschland und Europa als verhältnismäßig restriktiv. Computerprogramme "als solche" können bislang nicht patentiert werden. Das hat das nationale und das Europäische Patentamt in der Vergangenheit jedoch nicht daran gehindert, Tausende von Patenten für sogenannte computerimplementierte Erfindungen zu erteilen. Ein Versuch der EU-Kommission und des Ministerrates, diese Praxis mit Hilfe einer entsprechenden Direktive zu legalisieren, ist allerdings im Europäischen Parlament gescheitert (vgl. Haunss/ Kohlmorgen 2009; 2010).

Dies zeigt, dass es durchaus Kritik und Widerstand gegen die konkrete Ausgestaltung der Schutzrechte geistigen Eigentums, aber auch genereller gegen den „informationellen Kapitalismus“ gibt, der auf eine umfassende Kommodifizierung und Vermarktlichung neuen nützlichen Wissens zielt (Nuss 2006). Hier schließt das nächste Kapitel an, das jedoch nicht die Gegenbewegungen und deren Einfluss genauer betrachtet, sondern mit Blick auf einen Markthandel von Patenten untersucht, welche politischen und unternehmerischen Initiativen auf seine Förderung zielen und wo Grenzen insbesondere der Vermarktlichung als Handelsform von Patenten erreicht werden.

20 Wie die erwähnten Entscheidungen des US Supreme Court zeigen, hat hier nicht die Patentierungsmöglichkeit den Ausschlag für die Erfindungen gegeben, sondern die Erfinder haben zum Zwecke der besseren kommerziellen Nutzbarkeit ihrer Erfindungen die Patentierungsmöglichkeit gerichtlich gegen das Patentamt erstritten (vgl. NRC 2004: S. 21-28; auch Cullis 2007: S. 168-234). 
Schaubild 2: Patentanmeldungen USA, Japan, Deutschland und Europäisches Patentamt seit 1980 in Tsd.
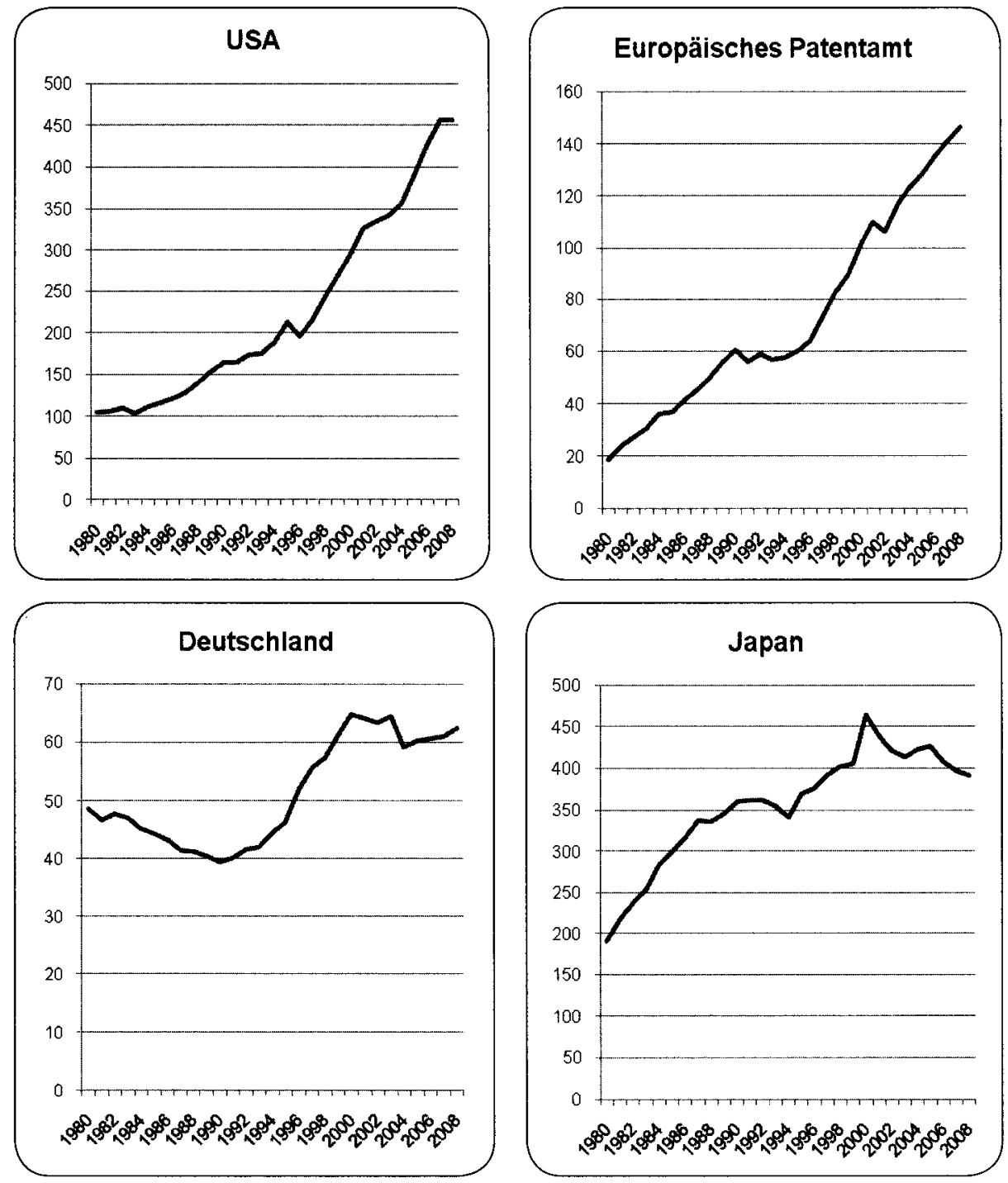

Quelle: Zuständige Patentämter und WIPO Patentstatistiken <http://www.wipo.int/ipstats/en/statistics/patents/> 


\section{Förderung des Patenthandels und Grenzen der Vermarktlichung}

Im Rahmen industriepolitischer und handelspolitischer Zielsetzungen soll die Politik der Stärkung und Erweiterung des Schutzes geistigen Eigentums mit Hilfe von Patenten in erster Linie Investitionen in neues nützliches Wissen schützen. Sie sichert damit die Monopolposition der Patentinhaber, die dann mit dem Handel von auf den Patenten basierenden Produkten einen wirtschaftlichen Gewinn erzielen können. Folgt man der Argumentation von Jessop (2007) im Anschluss an Polanyi, so geht es der marktgesteuerten kapitalistischen Wissensgesellschaft aber nicht nur um diesen Vorteil. Vielmehr zielt sie auf Effizienz bereits im Prozess des Entstehens einschließlich der Allokation neuen Wissens, die sie mit dem hier zu verankernden Marktprinzip gewährleisten will.

Wie in Kapitel II dargelegt stellen Patente eine grundsätzlich handelbare Form neuen technischen Wissens dar. Dabei sind verschiedene Handelsformen möglich. Die beiden hier besonders interessierenden sind der Verkauf und die Lizenzierung von patentiertem Wissen. Im Falle eines Verkaufs übernimmt der Käufer als neuer Eigentümer alle Rechte und Pflichten des Verkäufers. Häufiger als Kaufverträge sind aber Lizenzverträge. Hier überträgt der Patentinhaber das Recht der Nutzung des Wissens unter zu vereinbarenden Bedingungen und gegen Lizenzgebühren an einen oder mehrere Lizenznehmer. Viele Ökonomen halten starke Patente für notwendig, damit der Handel funktionieren und eine durch den Markt koordinierte Arbeitsteilung im Innovationsprozess realisiert werden kann (Hall/ Ziedonis 2001; Arora/ Merges 2004; Gambardella 2005; Ziedonis 2008). Sie sehen die Funktionsfähigkeit des Marktes in erster Linie durch schwache Schutzrechte beeinträchtigt. Ein starker Patentschutz, dies hat sich auch im historischen Rückblick angedeutet, mag eine notwendige Bedingung für einen funktionierenden Markt sein, hinreichend ist er jedoch nicht. Es bedarf offenbar zusätzlicher Maßnahmen und Initiativen, um die Marktentwicklung zu fördern. Einige politische Initiativen werden im Folgenden unter dem Aspekt analysiert, ob bzw. inwieweit sie Impulse für den Markthandel geben.

\subsection{Politische Initiativen}

Der Schwerpunkt der Analyse politischer Initiativen zur Förderung der Marktentwicklung liegt auf Deutschland. Betrachtet werden aber auch Japan und USA. Neben für den Patenthandel relevante bestehende gesetzliche Regelungen interessieren neuere politische Initiativen. Sie sind zumeist auf den Wissenstransfer von den Universitäten in die Unternehmen und den Zugang von kleinen und mittleren Unternehmen zum Patenthandel gerichtet.

Wie in den meisten Ländern war auch in Deutschland das politische Handeln lange Zeit auf Probleme der Erteilung, der Durchsetzung und des Schutzes von Patenten fokussiert. Aspekte ihrer externen, insbesondere marktmäßigen Verwer- 
tung wurden weitgehend vernachlässigt. ${ }^{21}$ Entsprechend wenige im Patentrecht verankerte Rahmenbedingungen zielen auf die Förderung des Patenthandels oder können zumindest eine entsprechende Wirkung haben.

Das Patentverfahren, Anmeldung und Prüfung, verursacht Kosten, in der Regel einige tausend Euro, die vom Anmeldenden zu tragen sind. Wird ein erteiltes Patent nicht regelmäßig aufrechterhalten, läuft der Schutz ab. Auch für die Aufrechterhaltung entstehen wieder Kosten, die progressiv steigen bis zur maximalen Laufzeit, die in den meisten Ländern 20 Jahre beträgt. Das Deutsche Patent- und Markenamt (DPMA) erhebt erstmalig für das dritte Jahr eine Gebühr in Höhe von 70 Euro für die Aufrechterhaltung eines Patents. Die für jedes weitere Jahr fällige Gebühr steigt bis auf 1.940 Euro für das 20. Patentjahr. Der Gesamtbetrag summiert sich auf rund 13.000 Euro. In USA liegt er niedriger, aktuell bei rund 7.500 Dollar. Die Gebühren können einen Anreiz darstellen, ungenutzte Patente zu veräußern. In diesem Zusammenhang ist auch die Regelung im deutschen Patentgesetz interessant $(\mathbb{2} 23)$, die vorsieht, dass die Aufrechterhaltungsgebühren halbiert werden, wenn Patentinhaber erklären, jedermann die Benutzung der Erfindung gegen angemessene Vergütung zu gestatten. Das DPMA veröffentlicht diese Lizenzbereitschaftserklärung. Auch diese Regelung kann als ein potentieller Anreiz verstanden werden, neues technisches Wissen Dritten zur Verfügung $\mathrm{zu}$ stellen. Schließlich sieht das Patentgesetz noch die Möglichkeit der Zwangslizenz vor (\$24). Mit Hilfe einer Zwangslizenz können Unternehmen ein Patent trotz wiederholter Ablehnungen seitens des Patentinhabers einlizenzieren. Diese Möglichkeit hat lediglich in der pharmazeutischen Industrie eine gewisse Bedeutung erlangt.

Während die genannten gesetzlichen Bestimmungen schon lange gelten und keinen aktiven Einfluss auf die Bereitschaft Patente zu handeln ausgeübt haben, lässt sich in jüngster Zeit eine Erweiterung des politischen Handlungsspektrums mit dem Ziel einer Förderung der externen Verwertung patentierten Wissens beobachten. Auf gesetzlicher Ebene sind vor allem die 2002 in Kraft getretenen Änderungen im Arbeitnehmererfindungsgesetz (ArbnErfG) und das neue Hochschulerfindungsrecht zu nennen. Das seit 1957 bestehende Gesetz über Arbeitnehmererfindungen gibt dem Arbeitgeber das Recht, patentfähige dienstliche Erfindungen der Arbeitnehmer in Anspruch zu nehmen, wenn diese im Gegenzug entschädigt werden (Bartenbach/Volz 2004). Das im Rahmen der Novellierung des ArbnErfG geänderte Hochschulerfinderrecht schafft das Hochschullehrerprivileg ab und räumt den Hochschulen das Recht ein, Erfindungen zu patentieren und wirtschaftlich zu verwerten (\$40-42). Den Erfindern steht pauschal ein Drittel der Lizenzeinnahmen nach Abzug der Patentierungskosten zu. Von dieser Regelung verspricht man sich einen verstärkten Wissenstransfer von den Hochschulen in die Wirtschaft und zusätzliche Einnahmen für die Hochschulen aus Patentlizenzen (Bundesministerium für Bildung und Forschung 2000).

21 Dies zeigt auch eine Rede, die Bundeskanzlerin Angela Merkel am 18. April 2007 anlässlich des „European Patent Forum“ des Europäischen Patentamts gehalten hat. Sie betont die Schutzfunktion der Patente und ihre Anreizfunktion für Innovationen und verweist dann erst darauf, dass sich der deutsche Innovationsrat mit der Frage der besseren Verwertung von Patenten und den Möglichkeiten, den „Patenthandel zu intensivieren“ beschäftigt (Merkel 2007). 
Im Zuge der Änderung des Hochschulerfindungsrechts wurden weitere innovations- und wirtschaftspolitisch motivierte Initiativen ergriffen, die sich an Hochschulen, Unternehmen und Erfinder richten und Fördermaßnahmen zur Optimierung der Verwertung von Patenten beinhalten. $\mathrm{Zu}$ den aktuellen Initiativen des Bundesministeriums für Wirtschaft und Technologie (2008) gehört das Programm SIGNO, das die schutzrechtliche Sicherung und die wirtschaftliche Verwertung von Forschungsergebnissen forcieren soll. Im Bereich der Universitäten sollen ganz überwiegend öffentlich finanzierte Patentverwertungsagenturen (PVA) eine bedeutende Rolle spielen. Die Universitäten bzw. ihre Technologietransferbüros sind in der Regel der übergeordneten Agentur des jeweiligen Bundeslandes zugeordnet, deren Dienste sie in Anspruch nehmen können (Kaiser/ Schillert 2005). Mit den PVAs trägt SIGNO der Tatsache Rechnung, dass Universitäten und öffentliche Forschungseinrichtungen nicht wie Unternehmen neues technisches Wissen intern wirtschaftlich nutzen können. Sie produzieren das Wissen in diesem Sinne immer auch für Dritte bzw. den Markt. Die PVAs sollen an Patentlizenzen interessierte Unternehmen finden, mit ihnen Verträge schließen und auf diese Weise Einnahmen generieren, die den Universitäten, den Erfindern und den Agenturen selbst zu Gute kommen. Das betriebswirtschaftliche Ergebnis der Verwertungsagenturen ist jedoch wenig beeindruckend. In den ersten fünf Jahren konnte keine Agentur auch nur annähernd kostendeckend arbeiten (Kienbaum 2006). Dies unterstreicht die generelle Problematik einer marktmäßigen Verwertung von Patenten.

SIGNO unterstützt auch kleine und mittelständische Unternehmen, die bisher wenig Erfahrung mit dem Patentieren haben, bei der Durchführung des Prozedere und der wirtschaftlichen Verwertung der Patente. Weitere Initiativen, wie der „InnovationMarket“, zielen auf die Etablierung eines frei zugänglichen InternetMarktplatzes für Erfindungen, welcher Innovationsanbieter, Kapitalgeber für Unternehmensgründer und etablierte Unternehmen zusammenbringen soll. Ausgewiesene SIGNO-Partner prüfen Inserate nach festgelegten Qualitätsstandards und übernehmen auf diese Weise die Rolle einer "third-party assistance“ (Williamson 1979), die als unabhängige und neutrale Experten Klarheit über den Wert einer Erfindung schaffen und verhindern sollen, dass minderwertige Patente die Entwicklung eines Marktes behindern. Gerade die Bewertung von Patenten ist sowohl für die Anbieter wie für die Nachfrager sehr schwierig. Sie bildet jedoch eine wichtige Voraussetzung für einen funktionierenden Handel (Razgaitis 2003, S. xvii). Eine erste Evaluation des Förderprogramms zeigt, dass es bisher auf die ohnehin schwachen Lizenzierungsaktivitäten der kleinen und mittelständischen Unternehmen „kaum Auswirkungen“ hat (Blind 2009: S. 102).

In USA markieren der Bayh-Dole Act und der Stevenson-Wydler Act aus dem Jahr 1980 eine Wende in der Patent- und Innovationspolitik mit dem expliziten Ziel einer ,increased commercialization of university inventions" (Mowery et al. 2004, S. 85). Bis dahin fehlte eine klare staatliche Regelung der Eigentumsverhältnisse an Erfindungen, die durch die öffentliche Hand gefördert wurden. Dies trug zusammen mit einer Reihe anderer Faktoren zu einer relativ niedrigen Kommerzialisierungsrate von staatlich geförderten Erfindungen bei. So hatte der US-amerikanische Staat im Jahr 1980 rund 28.000 Patente inne, wovon lediglich $5 \%$ an die Industrie auslizenziert waren (US Government Accounting Office 1998). Vor 1970 waren nur wenige 
zumeist private Universitäten in der Patentierung und Lizenzierung aktiv (Jaffe 2000). Erst seit Inkrafttreten des Bayh-Dole Act können Universitäten und nicht gewinnorientierte Forschungseinrichtungen Eigentumsrechte an Erfindungen erlangen. Zusammen mit einer erheblichen Lockerung der restriktiven Lizenzbestimmungen führten die gesetzlichen Änderungen zu einem starken Zuwachs von Patentanmeldungen und Lizenzverträgen (Hoppe/ Ozdenoren 2005).22 Zudem förderte die Einrichtung von Technologietransferbüros an Universitäten, wie im StevensonWydler Act vorgesehen, die Kommerzialisierung von Wissen aus der Forschung (Mowery, et al. 2001; kritisch Kenney 2008; auch Greenberg 2007, S.51-81).

Neben den genannten gesetzlichen Regeln finden wir weitere Programme, die Hilfen bei der Patentierung insbesondere von öffentlich finanzierten Erfindungen vorsehen. Sie sollen aber auch den Transfer patentierten Wissens aus Universitäten und Unternehmen, speziell kleinen und mittelständischen Firmen, unterstützen (siehe etwa Braunstein/ Plonsker o.J.). Wichtige Förderprogramme sind das „Small Business Innovative Research Program“ und das „Small Business Technology Transfer Program“. Die zuständige Small Business Administration (SBA) sponsert zahlreiche Beratungsleistungen für innovative Unternehmensgründer, wozu als Teilbereich auch die Patentierung und Kommerzialisierung von Erfindungen zählt (US Small Business Administration 2009).

Insgesamt lässt sich feststellen, dass politische Initiativen zur Förderung des Handels von Patenten in USA vor allem im Bereich des Transfers patentierten Wissens von den Universitäten in die Wirtschaft relativ erfolgreich sind. So erzielten die in der „Association of University Technology Managers (AUTM) “ zusammengeschlossenen Transferbüros von rund 160 Universitäten im Jahre $2007 \mathrm{Li}$ zenzeinnahmen von knapp 2,1 Mrd. US\$ (AUTM 2008). Das ist doppelt so viel wie im Jahre 2002..$^{23}$ Weitere politische Initiativen, auch solche des US Patentamts, zielen hauptsächlich auf den Patentschutz und nicht auf den Handel.

Ähnlich wie in USA und Deutschland liegt der Schwerpunkt der politischen Initiativen auch in Japan auf dem Wissenstransfer von den Universitäten in die Wirtschaft. So hat der 2002 in Kraft gesetzte Intellectual Property Basic Act einen deutlichen Schub in Richtung auf Patentierung und externe kommerzielle Patentverwertung von staatlich finanzierter Grundlagenforschung ausgelöst. In manchen Fällen verbleibt das Eigentumsrecht an einer Erfindung aus einer staatlichen Universität beim Erfinder, der das Patentrecht häufig nicht nur an die Universität, sondern auf informellem Wege auch an Unternehmen übergibt (Kneller 1999). ${ }^{24}$ Zumeist gelten die Erfindungen jedoch als nationale Erfindungen. Sie werden von der japanischen Wissenschafts- und Technologieagentur (JST), einer übergeordne-

22 Der Bayh-Dole Act reguliert unter anderem die Offenlegung einer Erfindung durch den Erfinder („Invention Disclosure“) gegenüber dem Technologietransferbüro der Forschungseinrichtung. Danach müssen Erfinder das Büro über eine Erfindung informieren. Viele Büros haben Standardprozeduren der Erfindungsmeldung etabliert.

23 Es handelt sich hier um Bruttoeinnahmen. Die Kosten für Forschung- und Entwicklung, für die Patentierung und für die Transferbüros sind nicht gegengerechnet.

24 Grundsätzlich müssen Erfinder ihre Erfindung gegenüber der Universität offenlegen, insbesondere wenn es sich um eine Erfindung mit militärischem Verwendungszweck handelt oder anderweitig ein nationales Interesse daran besteht (Kneller 1999). 
ten Verwertungsstelle für nationale Erfindungen, zum Patent angemeldet und auslizenziert. Mit ihrer Lizenzpolitik will die JST möglichst große technische Spillover-Effekte bewirken, weshalb sie bevorzugt nicht-exklusive Lizenzen vergibt (Japan Science and Technology Agency 2009; Kneller 1999). Neben der JST sind auch die Technologietransferbüros der Universitäten in der Auslizenzierung aktiv.

Ebenfalls vergleichbar mit USA und Deutschland sind japanische Förderaktivitäten für kleine und mittelgroße Unternehmen. Von besonderer Bedeutung ist hier das 2001 gegründete National Center for Industrial Property Information and Training (INPIT). INPIT ist eine unabhängige Verwaltungseinrichtung, die dem Japanischen Patentamt zugeordnet ist. Die Organisation fördert mit über hundert Außenstellen („,patent licensing advisors“) vor Ort die Lizenzierung von Patenten vor allem kleinerer und mittelgroßer Unternehmen. Im Vordergrund steht eindeutig die Patentverwertung und damit das Bemühen, lizenzierbare Technologien zu identifizieren und potentiellen Bedarf für sie zu ermitteln. Bis Anfang 2008 hat sich die Zahl der von INPIT angebahnten und erfolgreich abgeschlossenen Lizenzverträge auf rund 10.700 summiert. Der kumulierte "wirtschaftliche Effekt" (economic impact) dieser Lizenzverträge wird auf brutto etwas mehr als umgerechnet 2 Mrd. Euro bis Ende 2007 beziffert (INPIT 2008). Pro Vertrag sind das im Durchschnitt fast 200.000 Euro.

Zusammenfassend lässt sich festhalten, dass in den betrachteten Ländern politische Initiativen, die eindeutig auf eine Förderung des marktmäßigen Handels von Patenten zielen, selten sind. Am weitesten ging in Deutschland ein letztlich erfolgloser Antrag der FDP-Fraktion im Bundestag im März 2004. Die Bundesregierung wurde darin u.a. aufgefordert, "Regeln für die Bewertung von Urheberund Patentrechten zu erarbeiten " und - mit Blick auf den Handel besonders interessant - „die Grundlagen für eine Verbriefung von Urheber- und Patentrechten zu schaffen “ (Deutscher Bundestag, Drucksache 15/2863 vom 31.3.2004). In anderen Ländern und auf EU-Ebene ist es ähnlich. Fördermaßnahmen konzentrieren sich in erster Linie auf den Transfer von innovativem technischem Wissen aus Universitäten und öffentlichen Forschungseinrichtungen in die (möglichst einheimischen) Unternehmen. Erste gesetzliche Maßnahmen wurden hier in den frühen 1980er Jahren ergriffen und seit 2000 durch viele organisatorische Initiativen ergänzt und verstärkt. Erwähnenswert sind auch die deutschen und ähnliche japanische Bemühungen, mit dem „InnovationMarket" einen offenen Markt für Patente zu emulieren, auf dem (auch) Unternehmen ihre Patente anbieten können. Belastbare Daten zu dem Ergebnis dieser Initiativen liegen nicht vor. Insgesamt dominiert in der Politik weiterhin die Vorstellung, dass Patente in erster Linie dem Schutz des innovativen technischen Wissens der Unternehmen gegen „Produktpiraten" und "Trittbrettfahrer" dienen und es so ermöglichen, mit auf dem innovativen Wissen basierenden Produkten und Dienstleistungen Geld zu verdienen.25 Daher haben an kleine und mittelgroße Unternehmen adressierte Programme immer noch als erste Priorität, das Patentieren von Erfindungen und nicht deren externe marktmäßige oder nicht marktmäßige kommerzielle Verwertung zu fördern.

25 Ähnliches konstatiert Siegrist (2006: S. 79) für die Bedeutung des Urheberrechts. 


\subsection{Unternehmerische Initiativen}

Schaut man auf die Unternehmen, so finden sich einige Aktivitäten, die zwar auf den Patenthandel, nicht aber explizit auf die Schaffung eines Marktes für Patente zielen. Eine umfangreiche Umfrage in 2003/04 unter Inhabern von über 9.000 vom Europäischen Patentamt erteilten Patenten in sechs EU Staaten (DE, ES, FR, IT, NL, UK) zum Gebrauch ihrer Patente zeigt, dass insgesamt die externe Kommerzialisierung von Patenten die Ausnahme von der Regel darstellt (Tabelle 1). Danach wurden nur rund $13 \%$ aller Patente auslizenziert - und das nicht unbedingt immer gegen Bezahlung. Etwa $51 \%$ wurden ausschließlich intern genutzt. Während weitere knapp 19\% der Blockade von Wettbewerbern dienten, blieben über 17\% gänzlich ungenutzt (PatVal-EU Project - Gambardella 2005, S. 40; vgl. auch Blind et al. 2006). Gerade die letzten Zahlen werden als Indiz dafür betrachtet, dass großes Handelspotential existiert. Sie deuten aber auch an, dass Unternehmen nicht nur wie bereits angesprochen das Patenterteilungsverfahren, sondern auch das patentierte Wissen strategisch nutzen. Patentstrategien stellen für viele einen integralen Bestandteil ihrer Geschäftspolitik dar, wobei externe Kommerzialisierung keineswegs an erster Stelle steht (vgl. auch Fosfuri 2006).

\section{Tabelle 1: Nutzung von Patenten in der EU}

\begin{tabular}{lr}
\hline Exklusiv interne Nutzung & $50.5 \%$ \\
Lizenzierung & $6.4 \%$ \\
Kreuz-Lizenzierung & $3.0 \%$ \\
Lizenzierung und interne Nutzung & $4.0 \%$ \\
Blockade von Wettbewerbern & $18.7 \%$ \\
Ungenutzt & $17.4 \%$ \\
\hline & $(\mathrm{n}=7714)$
\end{tabular}

Quelle: PatVal-EU Project - Gambardella 2005, S. 40

Anders als kleine und mittelgroße (BDO 2007) verfügen speziell große Unternehmen über intern differenzierte Patentmanagementabteilungen, die das weltweite Patentgeschehen kontinuierlich beobachten (Gassmann/ Bader 2006, S. 198-314). Während das Patentieren in Zusammenarbeit mit externen Patentanwaltskanzleien und Beratern erfolgt, werden Aufgaben im Bereich des Technologietransfers und der Durchsetzung von Patenten intern von der Patentmanagementabteilung erledigt (vgl. Reiffenstein 2009). Das strategische Management des Patentportfolios eines großen Unternehmens umfasst auch die Veräußerung von technischem Wissen. Dies erfolgt aber nicht im engeren Sinne marktmäßig. Vielmehr stehen die Patentmanager und Licensing Executives der Großunternehmen oft in direktem Kontakt zueinander und benutzen diese Kontakte, um Transaktionen anzubahnen und zu realisieren (vgl. Lichtenthaler/ Ernst 2008a). In vielen Fällen wird das patentierte Wissen nicht wie eine Ware gehandelt, zumal, wie eingangs erwähnt (Kap II), vollständige Kommodifizierung oft nicht möglich ist. Patente dienen dann eher dem Tausch und der Kreuzlizenzierung von Schutzrechten und damit 
der Verhinderung von Schutzrechtsklagen der Wettbewerber (Bidault 1989; Hall/ Ziedonis 2001; Reitzig 2004). Auch wenn die Transaktion gegen Bezahlung erfolgt, muss das nicht bedeuten, dass nun der Erwerber das Patent nutzt, um auf dessen Basis ein neues Produkt herzustellen (Bidault 1989).

Damit Patente marktmäßig gehandelt werden können, müssen sie auch bewertet werden (Monk 2009, S. 478-481; vgl. auch Beckert 2009, S. 252-257). Eine von PricewaterhouseCoopers (2008) durchgeführte Befragung von Managern aus innovativen Unternehmen mit Sitz in Europa zeigt, dass Patentmanager Bewertungen am häufigsten im Rahmen der Pflege des Patentportfolios, der Festsetzung von Erfindervergütungen und der Steuerung und Kontrolle von Forschung und Entwicklung, also im Wesentlichen für interne Zwecke vornehmen. Demnach werden Patente durchaus als Vermögensgegenstände betrachtet, doch erfolgt ihre Bewertung nicht vordringlich mit Blick auf eine Vermarktung. Allerdings ist sie deshalb nicht unbedingt weniger schwierig und kann zudem je nach Verwendungspotential und internem Verwendungszweck des patentierten Wissens unterschiedlich ausfallen.

Viel stärker in Richtung auf die externe Kommerzialisierung von Patenten und die Schaffung von Märkten agieren Intermediäre. Solche Akteure, zu denen im Prinzip auch die erwähnten öffentlich finanzierten Patentverwertungsagenturen zur Förderung des Technologietransfers von der Wissenschaft in die Wirtschaft gehören, erbringen Dienstleistungen, die auch der Anbahnung und Realisierung von Patenttransaktionen dienen (Monk 2009, S. 472, 481). Die Intermediäre verfügen in der Regel über spezielles technisches, rechtliches und kommerzielles Know-how, und sie kennen auch Angebot und Nachfrage in bestimmten Branchen und Technikfeldern. In ihrer Rolle als relativ neutrale Dritte erhalten sie wichtige Informationen von den potenziellen Transaktionspartnern und können auf dieser Basis die Verwendungsmöglichkeiten und den Tauschwert eines Patents einschätzen. Häufig wenden sie Bewertungsmethoden an, die auf Erfahrungen und Daten aus früheren Transaktionen basieren und die sie gegenüber Wettbewerbern geheim halten (Troy/Werle 2008).

Intermediäre stellen kein völlig neuartiges Phänomen dar, wurden sie doch bereits als für den Patenthandel in USA in der zweiten Hälfte des 19. Jahrhunderts wichtige Akteure identifiziert (siehe Kap. III.1). Außer explorativen Studien für USA (z.B. Monk 2009; Auriol/ Felix 2009; auch Chesbrough 2006) gibt es allerdings speziell für Deutschland keine aktuellen Untersuchungen zu Struktur und Aktivitäten dieser Gruppe (vgl. aber Tietze/ Herstatt 2009). Eigene Recherchen in Unternehmensdatenbanken waren mit großen Schwierigkeiten konfrontiert, weil es in den standardisierten Klassifikationen keine eigene Kategorie gibt, in die die Intermediäre eingeordnet werden könnten. Eine genauere Durchsicht der Unternehmensinformationen in verschiedenen Datenbanken ermöglichte schließlich die Identifikation von zunächst 94 Intermediären, zu deren Geschäftsfeldern (im Jahre 2008) auch die Patentverwertung gehört. Eine vorläufige Auswertung der verfügbaren Informationen zeigt, dass gut ein Drittel dieser Unternehmen nach dem Jahr 2000 gegründet wurde und die meisten sehr klein sind. Häufig sind es Einzelpersonen, die ihre Dienste anbieten. Rund ein Drittel ist auch in der Vermögensberatung tätig, $14 \%$ bieten zudem Rechts- und Steuerberatung an. Die Daten las- 
sen keine sicheren Rückschlüsse darauf zu, wie sich die Zahl der Intermediäre entwickelt hat. Sie sprechen jedoch dafür, dass deren Zahl wächst, es aber gleichzeitig wenig erfolgversprechend ist, sich vollständig auf die Tätigkeit als Makler für Patente zu spezialisieren.

Die von Unternehmen und anderen nichtstaatlichen Akteuren eher unkoordiniert vorangetriebene Entwicklung eines Marktes für Patente befindet sich in einer „trial and error“ Phase mit unsicherem Ausgang. Speziell Intermediäre experimentieren mit unterschiedlichen Modellen einer marktmäßigen Transaktion von Patenten. Hierzu gehören Internet-Plattformen für den online-Verkauf von Patenten ebenso wie Patentauktionen und Patentfonds. Die Erfolge dieser Aktivitäten sind jedoch begrenzt (vgl. hierzu Malackowski 2006; Lipfert/ Ostler 2007; Lichtenthaler/ Ernst 2008b; Tietze/ Herstatt 2009). Kollektive koordinierte Aktionen von Unternehmen und Verbänden zur Schaffung eines Marktes, wie sie in anderen innovativen Bereichen zu beobachten sind (vgl. Möllering 2009; 2010), fehlen für Patente weitgehend. So setzen Beobachter der Entwicklung, soweit sie bereits von einem „Emerging Patent Marketplace“ sprechen, vor allem auf die Intermediäre, die aus ihrer Sicht entscheidend zur Marktentwicklung beitragen, ohne dass sie nennenswerte politische Unterstützung finden (Auriol/ Felix 2009). Gleichzeitig wird auf die als handelsfördernd betrachtete zunehmende Wahrnehmung von $\mathrm{Pa}$ tenten als Vermögensgegenständen verwiesen. Hierfür sprechen die Ergebnisse einer von uns vorgenommenen Auswertung von inzwischen über $1.700 \mathrm{im}$ Social Science Citation Index des ISI Web of Knowledge erfassten Fachzeitschriften (Tabelle 2). Die Zahl der in den Zeitschriften erschienenen Artikel, die die Themen Patentbewertung, Patenthandel oder Patentmanagement behandeln, stieg für die drei Bereiche zusammen von 50 im Zeitraum 1980 bis 1989 auf 854 in den Jahren 2000 bis 2009. Allein die Problematik der Patentbewertung als einer Voraussetzung für den Patenthandel wurde von 2000 bis 2009 in 306 Artikeln diskutiert. Im Zeitraum 1980 bis 1989 waren es nur 11. Die Publikationen erfolgten in der Regel in Wirtschafts- und Managementjournalen. Deren Zahl ist zwar seit 1980 auch gestiegen, doch dürfte dies eher trendverstärkend wirken.

Tabelle 2: Zahl der Veröffentlichungen von Artikeln zu Patentbewertung, Patenthandel und Patentmanagement in etablierten Fachzeitschriften*

\begin{tabular}{lccc}
\hline Zeitraum & 1980-1989 & 1990-1999 & 2000-2009 \\
\hline $\begin{array}{l}\text { Suchbegriff Patent: } \\
\text { Value }\end{array}$ & 11 & 71 & 306 \\
$\begin{array}{l}\text { Value, or Trade, } \\
\text { or Management }\end{array}$ & 50 & 266 & 854 \\
\hline
\end{tabular}

*Recherche im Social Science Citation Index des ISI Web of Knowledge mit über 1.700 Journals (Stand: 31.03.10). Durchsucht wurden die Titel und Abstracts der Artikel, und es wurden Plausibilitätskontrollen durchgeführt. 


\subsection{Umfang des Patenthandels}

Untersuchungen und Daten zum Patenthandel sind Mangelware. Die vorliegenden Studien zeigen, dass zwar ein nennenswerter Handel mit Patenten stattfindet, das Potenzial aber bei weitem nicht ausgeschöpft wird. Die Überzeugung, dass die "markets for technology" groß sind und weiter wachsen werden, ist besonders unter Ökonomen weit verbreitet (Arora et al. 2001). Schätzungen und vor allem Prognosen (z.B. Rivette/ Kline 2000) sind jedoch oft überzogen und leicht angreifbar (Bessen/ Meurer 2008, S. 112-119). Guellec und van Pottelsberghe de la Potterie $(2007$, S. 91, 92) vergleichen unterschiedliche Schätzungen der Größe des Marktes für Lizenzen aller Art und kommen zu dem Ergebnis, dass in der Mitte des Jahrzehnts der amerikanische Markt einen Umfang von 40 bis $50 \mathrm{Mrd}$. US\$ hatte und der europäische und der japanische Markt eine Größe von jeweils 15 bis $20 \mathrm{Mrd}$. US\$ aufwiesen. Basierend auf einer Auswertung von Informationen aus der Datenbank des US Patentamts für den Zeitraum von 1980 bis 2001 kommt Serrano (2006) zu dem Ergebnis, dass ungefähr 20\% aller US Patente kleinerer innovativer Firmen mindestens einmal an andere Firmen verkauft wurden. Über das Handelsvolumen insgesamt macht er aber keine Aussagen. Auch beschränkt er sich auf die Übertragung von Patenten und berücksichtigt keine Transaktionen in Form von Lizenzierungen, obwohl diese insgesamt häufiger erfolgen.

Wegen der Vertraulichkeit privater Verträge und einer fehlenden rechtlichen Verpflichtung, die Einkünfte aus entsprechenden Transaktionen offen zu legen, gibt es kaum belastbare Daten zum finanziellen Volumen des Patenthandels (Kamiyama et al. 2006, S. 16). Die Autoren der erwähnten Umfrage unter 9000 Patentinhabern in der EU schätzen auf der Basis von Wertangaben der Befragten zu ihren Lizenzgeschäften, dass der europäische Markt für Patente im Jahr 2002 eine Größenordnung von 15,6 Mrd. Euro hatte. Er hätte nach ihren Schätzungen aber um fast $70 \%$ größer sein können, wenn weitere $7 \%$ der Befragten, die bereit waren, ihre Patente zu lizenzieren, Lizenznehmer gefunden hätten, was ihnen offenbar nicht gelungen war (Gambardella et al. 2007, S. 1164). Dies sind allerdings Schätzungen auf der Basis von Befragungsdaten und keine direkten Informationen über tatsächlich erfolgte Transaktionen.

Solche Informationen gibt es in aggregierter Form zum internationalen Lizenzhandel. Sie werden von vielen OECD Ländern erhoben. Allerdings umfassen die Daten in einigen Ländern auch Lizenzen im Bereich des Urheberrechts und der Warenzeichen (Marken). Die Einnahmen aus der internationalen Lizenzierung all dieser Schutzrechte wuchsen weltweit von $10 \mathrm{Mrd}$. US\$ im Jahre 1985 auf fast 110 Mrd. US\$ im Jahre 2004 (Kamiyama et al. 2006, S. 17, 18). Deutschland verdoppelte zwischen 1990 und 2003 seine Einnahmen aus internationalen $\mathrm{Pa}$ tentlizenzen und Patentverkäufen von 1,3 auf 2,7 Mrd. Euro (Kamiyama et al., S. 18). Sie sind neueren Zahlen der Bundesbank zufolge bis zum Jahre 2007 auf 3,6 Mrd. Euro gestiegen, nachdem sie 2005 allerdings bereits über 4 Mrd. Euro lagen (Deutsche Bundesbank 2008, S. 15). Die schon erwähnten Daten der Weltbank (Schaubild 1) lassen erkennen, dass die internationalen Zahlungen von Lizenzgebühren zwischen 1980 und 2007 ganz erheblich zugenommen haben. Doch darf man die Entwicklung auch nicht überbewerten. Für USA, das bei weitem die 
höchsten Einkünfte hat, liegt der Anteil dieser Einkünfte am Wert der Exporte von Dienstleistungen seit einigen Jahren bei ungefähr $16 \%$. Bezogen auf alle Exporte beträgt der Anteil 5\%. Von diesen Einkünften dürfte maximal die Hälfte aus Patentlizenzen stammen (vgl. Park/ Lippoldt 2004, S. 15, 28).

Bemerkenswert an den Zahlen für den internationalen Handel ist, dass sie zu einem großen Teil aus Transaktionen stammen, die zwischen verbundenen Unternehmen stattfinden. Im Jahre 2005 lag dieser Anteil in den OECD Staaten deutlich über 50\% (Kamiyama et al. 2006, S. 19). In Deutschland überschritt er sogar 90\% (Deutsche Bundesbank 2008, S. 23). Solche Lizenzaktivitäten kann man aber, wie bereits erwähnt, nicht als Markthandel bezeichnen, und es ist fraglich, ob man überhaupt von externen Transaktionen sprechen kann. Auch Studien auf Unternehmensebene zum Lizenzierungsverhalten zeigen, dass die Firmen ihre Transaktionen ganz bevorzugt auf Partner beschränken, mit denen sie schon in der Vergangenheit Geschäftsbeziehungen unterhielten (Bidault/ Fischer 1994, S. 373; vgl. auch Anand/ Khanna 2000, S. 114 ff.).

\subsection{Handel ohne Markt}

Die verfügbaren Daten lassen den Schluss zu, dass von einem gut funktionierenden Markt für Patente nicht die Rede sein kann. Betrachtet man die Marktwirtschaft als eine "Wirtschaftsform, die nur fertige Güter und Dienstleistungen“ (Deutschmann 2007, S. 90), also solche Produkte tauscht, die für den Markttausch produziert wurden, dann kann das nicht überraschen. Der Handel mit Patenten ist geprägt von strategischen und fundamentalen Unsicherheiten, die hier nicht im Einzelnen dargestellt werden können (Troy/ Werle 2008; vgl. auch Geroski 1995, S. 91-93).

Wichtig ist, dass Patente häufig ein Ergebnis von Forschungs- und Entwicklungsarbeiten darstellen, deren Umsetzung in konkrete marktfähige Produkte nicht erfolgt (ist). Forschungs- und Entwicklungsprozesse zeichnen sich dadurch aus, dass die beste Vorgehensweise selten offensichtlich ist, das Ergebnis nie perfekt auf der Basis des Inputs vorhergesagt werden kann und das Resultat einschließlich seiner Neuheit grundsätzlich unsicher ist (Machlup 1984, S.169; Arrow 1985, S. 111; Van de Ven et al. 1999, S. 170). Mit dieser Unsicherheit ist zwar in erster Linie der Anbieter patentierten Wissens konfrontiert, doch auch der Kaufinteressent kann nicht sicher sein, dass das zu erwerbende Wissen ausreicht, um auf seiner Basis ein neues Produkt zu entwickeln. Zwischen dem Anbieter und dem potentiellen Käufer bestehen in der Regel deutliche Informationsasymmetrien. Das Wissen des Patentinhabers übersteigt den Inhalt dessen, was in der Patentschrift niedergelegt ist, während der potentielle Käufer kaum Informationen besitzt, die es ihm ermöglichen würden, die „Qualität“ eines Patents zu beurteilen (Bessen 2006, S. 22). Derartige Informationsasymmetrien sind eine Quelle von Unsicherheit, auch wenn der Patentinhaber nicht absichtlich Wissen zurückhält. Sie deuten an, dass schon wegen seiner eingeschränkten Kodifizierbarkeit (siehe oben Kap. II) die vollständige Appropriation von Wissen durch Dritte erheblich eingeschränkt oder sogar unmöglich ist (Arrow 1985; Machlup 1984, S. 182185). Das Patent allein enthält also selten alle Informationen, die zur Nutzung des 
Wissens und damit auch zu dessen erfolgreichem Handel erforderlich sind. Dies macht es aus der Sicht des Käufers oft erforderlich, komplementäres Know-how vom Patentinhaber zu erwerben (Clarkson 2001, S. 6).

Neues technisches Wissen ist also oft nur unvollständig kommodifizierbar. Damit wird es unwahrscheinlich, dass sich der Handel von Patenten als punktueller Markttausch zwischen anonymen Parteien vollzieht, auch wenn die Patente rechtlich klar definiert und gut abgesichert sind, was allerdings in vielen Technikfeldern nicht der Fall ist (Bessen/ Meurer 2008, S. 73-94). Vielmehr sind die Partner, was sich aus den Daten zum internationalen Handel und den Untersuchungen zum Lizenzierungsverhalten von Unternehmen ablesen lässt, häufig einander zumindest gut bekannt oder rechtlich verbunden. In solchen Beziehungen besteht oft bereits eine Vertrauensbasis, die es den Transaktionspartnern erleichtert, gegenseitige, auch informelle Verpflichtungen einzugehen, die zum beiderseitigen Nutzen beitragen. ${ }^{26}$ Für einander weniger bekannte Parteien dienen Lizenzverträge, über die oft monatelang verhandelt wird, als präferiertes rechtliches Instrument, Unsicherheit zu bewältigen (vgl. Brousseau et al. 2007; Troy/ Werle 2008). In den Verträgen können neben den Modalitäten der Zahlung von Lizenzgebühren der Transfer von zusätzlichem Know-how und eine Zusammenarbeit bei der Nutzung und zukünftigen Weiterentwicklung der transferierten Innovation vereinbart werden (vgl. Arora 1995; Burhop/ Lübbers 2009). So markiert der Transfer neuen Wissens den Beginn einer längerfristigen Zusammenarbeit zwischen den Handelspartnern, sofern diese, was häufig der Fall ist, nicht ohnehin bereits besteht. Die Zusammenarbeit basiert auf privat gesetzten Regeln. Politische Vorgaben existieren praktisch nicht. Patenthandel findet also statt, er erfolgt aber eher in kooperativer als in marktmäßiger Form. Die geschlossenen Verträge sind oft Kooperationsverträge und keine einfachen Kaufverträge. Dies ist zwar auch darauf zurückzuführen, dass die rechtlichen und politischen Rahmenbedingungen kaum Anreize für den Markthandel bieten. Mindestens ebenso wichtig sind aber die Schwierigkeiten der Kodifizierung und Kommodifizierung des Wissens.

\section{Konklusion}

Die Wissensgesellschaft, so die neoliberale Position, funktioniert erst dann ökonomisch effizient, wenn innovatives technisches Wissen nicht nur patentrechtlich geschützt, sondern auch marktmäßig gehandelt wird. Während die historische Entwicklung des Patentsystems vorwiegend wegen der unterschiedlichen industriepolitischen und handelspolitischen Strategien der Staaten insgesamt uneinheitlich verlaufen ist, gewinnt mit dem Beginn der Pro-Patent-Ära in den 1980er Jahren der Prozess der internationalen Angleichung der Patentsysteme an Dynamik. Gleichzeitig werden die Möglichkeiten der Patentierung, zunächst in USA und später - in unterschiedlicher Intensität - weltweit erweitert. Länder mit hohem Exportvolumen und starken High-Tech Branchen sind die treibenden Kräfte. Sie

26 Lizenzverträge zwischen rechtlich verbundenen Unternehmen (Mutter- und Tochterfirmen) dienen nicht unbedingt in erster Linie der Übertragung patentierten Wissens, sondern werden etwa aus steuerlichen Gründen geschlossen. 
werden ihrerseits angetrieben von großen multinationalen Konzernen, die sich insbesondere gegen „Produktpiraten“ und „Trittbrettfahrer“ schützen wollen. Die politischen Maßnahmen zur Erweiterung und Stärkung von Patenten zielen bis in die jüngere Vergangenheit ganz überwiegend auf diesen Schutz der Exportwirtschaft, indem sie die Etablierung weltweiter Monopole auf der Basis international harmonisierter Patentregeln begünstigen. Letztlich geht es bei all diesen Maßnahmen um die Sicherung nationaler wirtschaftlicher Vorteile durch das Patentsystem. Die Patentpolitik ist also viel stärker neomerkantilistisch als neoliberal geprägt.

Entsprechend sind politische Initiativen, die direkt auf die Förderung des Handels patentierten Wissens als solchem zielen, wie der Blick auf Deutschland, Japan und USA zeigt, eher selten. In erster Linie sollen Patente den kontrollierten Wissenstransfer von Universitäten und öffentlichen Forschungseinrichtungen zu den Unternehmen unterstützen. Da das Patentrecht nur wenige Regeln enthält, die tendenziell den Markttausch fördern, und auch die Patentpolitik hier wenig bewirkt, sind es die potentiellen Marktteilnehmer selbst, die den Markt zu entwickeln und zu gestalten versuchen. Besondere Bedeutung erlangen Intermediäre, die als Makler agieren und mit unterschiedlichen marktorientierten Formen des Handels von geistigem Eigentum experimentieren. Aber auch die Intermediäre sind mit den grundsätzlichen Schwierigkeiten konfrontiert, die sich aus der zumindest nicht vollständigen Kommodifizierbarkeit von Wissen ergeben (Mokyr 2002, S. 22). Doch nicht nur der Umstand, dass größere Teile des neuen Wissens nicht für den Markt produziert werden, sondern auch die im Zusammenhang mit dem Wissenstransfer zu bewältigenden Unsicherheiten lassen erwarten, dass auch in Zukunft Patente in der Regel nicht auf Märkten gehandelt werden. Die vorliegenden Daten zum Patenthandel weisen in diese Richtung. Wenn also patentiertes Wissen überhaupt gehandelt wird, geschieht das typischerweise in kooperativer Form. Konstruktivistisch ausgedrückt entsteht erst im Prozess der Kooperation das zu handelnde Wissensgut.

\section{Literatur}

Anand, Bharat N., und Tarun Khanna. 2000. The Structure of Licensing Contracts. The Journal of Industrial Economics 48: 103-135.

Arora, Ashish. 1995. Licensing Tacit Knowledge. Intellectual Property Rights and the Market for Know-How. Economics of Innovation and New Technology 4: 41-60.

Arora, Ashish, und Robert P. Merges. 2004. Specialized supply firms, property rights and firm boundaries. Industrial and Corporate Change 13: 451-475.

Arora, Ashish, Andrea Fosfuri, und Alfonso Gambardella. 2001. Markets for Technology. The Economics of Innovation and Corporate Strategy. Cambridge, MA: MIT Press.

Arrow, Kenneth. 1985. Economic Welfare and the Allocation of Resources for Invention.

In Production and Capital, Hrsg. Kenneth Arrow, 104-119. Cambridge, MA: The

Belknap Press of Harvard University Press.

Auriol, Laudeline, and Bernard Felix. 2009. The Emerging Patent Marketplace. STI Working Paper 2009/9. Paris: OECD.

Aspers, Patrik. 2009. How are markets made? MPIfG Working Paper 09/2. Cologne: Max Planck Institute for the Study of Societies. 
AUTM (The Association of University Technology Managers). 2008. U.S. Licensing Activity Survey: FY2007. Survey Summary. Deerfield, IL: AUTM. http://www.autm.net/AM/ Template.cfm?Section=FY_2007_Licensing_Activity_Survey\&Template=/CM/ContentDisplay.cfm\&ContentID=2805.

Barben, Daniel. 2007. Politische Ökonomie der Biotechnologie. Innovation und gesellschaftlicher Wandel im internationalen Vergleich. Frankfurt a. M.: Campus.

Bartenbach, Kurt, und Franz-Eugen Volz. 2004. Arbeitnehmererfindungen: Praxisleitfaden mit Mustertexten. 3. Auflage. Köln: Carl Heymanns.

BDO Deutsche Warentreuhand. 2007. Studie zum Thema „Bewertung von Patenten“. Hamburg: BDO.

Beckert, Jens. 2009. The social order of markets. Theory and Society 38: 245-269.

Bessen, James. 2006. The Value of U.S. Patents by Owner and Patent Characteristics. Working Paper Series, Law and Economics, Working Paper 06-46. Boston: Boston University School of Law.

Bessen, James, und Michael J. Meurer. 2008. Patent Failure. How Judges, Bureaucrats, and Lawyers Put Innovators at Risk. Princeton: Princeton University Press.

Bidault, Francis. 1989. Technology Pricing: From Principles to Strategy. New York: St. Martin's.

Bidault, Francis, und William A. Fischer. 1994. Technology transactions: networks over markets. RóD Management 24: 373-386.

Blind, Knut. 2009. Die volkswirtschaftliche Bedeutung geistigen Eigentums und dessen Schutz mit Fokus auf den Mittelstand. Forschungsbericht Nr. 579 des Bundesministeriums für Wirtschaft und Technologie. Berlin: Bundesministerium für Wirtschaft und Technologie.

Blind, Knut, Jakob Edler, und Michael Friedewald. 2003. Geistige Eigentumsrechte in der Informationsgesellschaft: Eine Analyse der Rolle gewerblicher Schutzrechte bei Gründung und Markteintritt sowie für die Innovations- und Wettbewerbsfäbigkeit von Softwareunternehmen anhand unternebmens- und softwarebezogener Fallstudien. Karlsruhe: Fraunhofer Institut für System- und Innovationsforschung.

Blind, Knut, et al. 2006. Motives to patent. Empirical evidence from Germany. Research Policy 35: 655-672.

Boldrin, Michele, und David Levine. 2002. The Case against Intellectual Property. The American Economic Review 92: 209-212.

Braithwaite, John, und Peter Drahos. 2000. Global Business Regulation. Cambridge, UK: Cambridge University Press.

Brand, Andreas, und Ursula Holtgrewe. 2010. KDE im Kontext: Open Source Software Entwicklung und öffentliche Güter. In Wissensökonomie und Innovation, Hrsg. Manfred Moldaschl und Nico Stehr, 371-400. Marburg: Metropolis.

Brand, Ulrich, et al. 2008. Conflicts in Environmental Regulation and the Internationalisation of the State. Contested Terrains. London/New York: Routledge.

Braunstein, D., und Larry Plonsker. o. J: The Licensing Decision. Prepared for the US Department of Energy. Washington: US Department of Energy.

Brousseau, Eric, Régis Coeurderoy, und Camille Chaserant. 2007. The Governance of Contracts. Empirical Evidence on Technology Licensing Agreements. Journal of Institutional and Theoretical Economics 163: 205-235. 
Bundesministerium für Bildung und Forschung. 2000. Begründung zum Änderungsvorschlag für $\mathbb{S} 42$ Arbeitnehmererfindungsgesetz der Bund-Länder-Kommission für Bildungsplanung und Forschungsförderung (BLK). Berlin. http://www.bmbf.de/_media/press/BLK3010.pdf.

Bundesministerium für Wirtschaft und Technologie. 2008. Signo Deutschland. http://www. signo-deutschland.de/content/index_ger.html.

Burhop, Carsten. 2009. The Transfer of Patents in Imperial Germany. Preprints 2009/26. Bonn: Max Planck Institute for Research on Collective Goods.

Burhop, Carsten, und Thorsten Lübbers. 2009. The Historical Market for Technology Licenses. Chemicals, Pharmaceuticals, and Electrical Engineering in Imperial Germany. Preprints 2009/25. Bonn: Max Planck Institute for Research on Collective Goods.

Chesbrough, Henry. 2006. Emerging Secondary Markets for Intellectual Property. US and Japan Comparisons. Lafayette, CA: Open Innovation Corporation.

Clarkson, Gavin. 2001. Avoiding suboptimal Behaviour in Intellectual Asset Transactions. Economic and Organizational Perspectives on the Sale of Knowledge. Discussion Paper 330. Cambridge, MA: Harvard Law School.

Cohen, Wesley M. 2005. Patents and Appropriation: Concerns and Evidence. Journal of Technology Transfer 30: 57-71.

Cohen, Wesley M., Richard R. Nelson, und John P. Walsh. 2000. Protecting Their Intellectual Assets. Appropriability Conditions and Why U.S. Manufacturing Firms Patent (or Not). NBER Working Paper 7552. Cambridge, MA: The National Bureau of Economic Research.

Coriat, Benjamin, und Oliver Weinstein. 2008. IPR Regimes, Firms and the Commodification of Knowledge. CEPN Working Paper 13-2008. Paris: Centre d'Economie de l'Université Paris Nord.

CSTB, National Research Council, Computer Science and Telecommunications Board, und Committee on Intellectual Property Rights in the Emerging Information Infrastructure. 2000. The Digital Dilemma. Intellectual Property in the Information Age. Washington, D.C.: The National Academies Press.

Cullis, Roger. 2007. Patents, Inventions and the Dynamics of Innovation. Cheltenham: Edward Elgar.

Dasgupta, Partha, und Paul A. David. 1993. Toward a new economics of science. Research Policy 23: 487-521.

David, Paul A. 1993. The Evolution of Intellectual Property Institutions. Working Paper 93-009. Maastricht: MERIT (Maastricht Economic Research Institute on Innovation and Technology).

David, Paul A. 1998. Common Agency Contracting and the Emergence of "Open Science" Institutions. The American Economic Review 88: 15-21.

David, Paul A., und Dominique Foray. 2002. An introduction to the economy of the knowledge society. International Social Science Journal 171: 9-23.

Deutsche Bundesbank. 2008. Technologische Dienstleistungen in der Zahlungsbilanz. Frankfurt a. M.: Deutsche Bundesbank.

Deutschmann, Christoph. 2007. Unsicherheit und soziale Einbettung. Konzeptionelle Probleme der Wirtschaftssoziologie. In Märkte als soziale Strukturen, Hrsg. Jens Beckert, Rainer Diaz-Bone und Heiner Ganßmann, 79-93. Frankfurt a. M.: Campus. 
Djelic, Marie-Laure. 2006. Marketization: From intellectual agenda to global policy-making. In Transnational Governance. Institutional Dynamics of Regulation, Hrsg. MarieLaure Djelic und Kerstin Sahlin-Andersson, 53-73. Cambridge: Cambridge University Press.

Dolata, Ulrich. 2008. Das Internet und die Transformation der Musikindustrie. Rekonstruktion und Erklärung eines unkontrollierten Wandels. Berliner Journal für Soziologie 18: 339-519.

Dolata, Ulrich. 2009. Technological innovations and sectoral change. Transformative capacity, adaptability, patterns of change: An analytical framework. Research Policy 38: 1066-1076.

Drahos, Peter. 2005. Intellectual property rights in the knowledge economy. In Handbook on the Knowledge Economy, Hrsg. David Rooney, Greg Hearn und Abraham Ninan, 139-151. Cheltenham: Edward Elgar.

Drucker, Peter F. 1993. Post-Capitalist Society. New York: HarperCollins.

Drucker, Peter F. 2007. The Essential Drucker. Oxford: Elsevier.

Dutfield, Graham. 2003. Intellectual Property Rights and the Life Science Industries. A Twentieth Century History. Aldershot: Ashgate.

Eckl, Julian. 2004. Die politische Ökonomie der "Wissensgesellschaft". Geistige Eigentumsrechte und die Frage des Zugangs zu Ideen. Marburg: Tectum.

Eimer, Thomas R. 2007. Regime Failure and Bilaterialization. The Millenium Goals and the Substantive Patent Law Treaty. 4th ECPR General Conference. PN 445: The Politics of Intellectual Property. Pisa, Italy (6-8 September 2007).

Eimer, Thomas R. 2008. Decoding Divergence in Software Regulation: Paradigms, Power Structures, and Institutions in the United States and the European Union. Governance 21: 275-296.

European Patent Office. 2007. Scenarios for the Future. München: European Patent Organisation

Fligstein, Neil. 2001. The architecture of markets. An economic sociology of twenty-firstcentury capitalist societies. Princeton, NJ: Princeton University Press.

Foray, Dominique. 2004. Economics of Knowledge. Cambridge, MA: MIT Press.

Fosfuri, Andrea. 2006. The Licensing Dilemma: Understanding the determinants of the rate of technology licensing. Strategic Management Journal 27: 1141-1158.

Fourcade, Marion. 2009. Economists and Societies. Discipline and Profession in the United States, Britain, and France, 1890 s to 1990s. Princeton: Princeton University Press.

Gambardella, Alfonso. 2005. Patents and the division of innovative labor. Comment on "Specialized supply firms, property rights, and firm boundaries" by Ashish Arora and Robert Merges. Industrial and Corporate Change 14: 1223-1233.

Gambardella, Alfonso, Paola Giuri, und Allessandra Luzzi. 2007. The markets for patents in Europe. Research Policy 36: 1163-1183.

Gassmann, Oliver, und Martin A. Bader. 2006. Patentmanagement. Innovationen erfolgreich nutzen und schützen. Berlin: Springer.

Geroski, Paul. 1995. Markets for Technology. Knowledge, Innovation and Appropriability. In Handbook of the Economics of Innovation and Technological Change, Hrsg. Paul Stoneman, 90-131. Oxford: Blackwell.

Granstrand, Ove. 2000. The Economics and Management of Intellectual Property. Towards Intellectual Capitalism. Cheltenham: Edward Elgar. 
Greenberg, Daniel S. 2007. Science for Sale. Chicago: University of Chicago Press.

Guellec, Dominique, und Bruno van Pottelsberghe de la Potterie. 2007. The Economics of the European Patent System. IP Policy for Innovation and Competition. Oxford: Oxford University Press.

Hall, Bronwyn H. 2005. Exploring the Patent Explosion. Journal of Technology Transfer 30: 35-48.

Hall, Bronwyn H., und Rosemarie Ham Ziedonis. 2001. The patent paradox revisited. An empirical study of patenting in the U.S. semiconductor industry, 1979-1995. RAND Journal of Economics 32: 101-128.

Harison, Elad. 2008. Intellectual Property Rights, Innovation and Software Technologies. The Economies of Monopoly Rights and Knowledge Disclosure. Cheltenham: Edward Elgar.

Harvey, Mark, und Andrew McMeekin. 2007. Public or Private Economies of Knowledge. Cheltenham: Edward Elgar.

Haunss, Sebastian, und Lars Kohlmorgen. 2009. Lobbying or politics? Political claimsmaking in IP conflicts. In The Politics of Intellectual Property, Hrsg. Sebastian Haunss und Kenneth C. Shadlen, 107-128. Cheltenham: Edward Elgar.

Haunss, Sebastian, und Lars Kohlmorgen. 2010. Conflicts about intellectual property claims. The role and function of collective action networks. Journal of European Public Policy 17: 242-262.

Hofmann, Jeanette, und Christian Katzenbach. 2006. Einführung. In Wissen und Eigentum. Geschichte, Recht und Ökonomie stoffloser Güter, Hrsg. Jeanette Hofmann, 9-20. Bonn: Bundeszentrale für politische Bildung.

Höpner, Martin, et al. 2009. Liberalisierungspolitik. Eine Bestandsaufnabme von zweieinhalb Dekaden marktschaffender Politik in entwickelten Industrieländern. MPIfG Discussion Paper 09/7. Köln: Max-Planck-Institut für Gesellschaftsforschung.

Hoppe, Heidrun C., und Emre Ozdenoren. 2005. Intermediation in innovation. International Journal of Industrial Organization 23: 483-503.

Howells, Jeremy. 1996. Tacit Knowledge, Innovation and Technology Transfer. Technology Analysis \& Strategic Management 8: 91-106.

INPIT (National Center for Industrial Property Information and Training). 2008. Guide to INPIT's Measures for Encouraging Patent Licensing. Tokyo: INPIT. http://www.ryutu. inpit.go.jp/en/pdf/guide2009.pdf.

Jaffe, Adam B. 2000. The US patent system in transition. Policy innovation and the innovation process. Research Policy 29: 531-557.

Jaffe, Adam B., und Josh Lerner. 2004. Innovation and its Discontents. Princeton: Princeton University Press.

Japan Science and Technology Agency. 2009: Content of Activities. http://www.jst.go.jp/ EN/about/content.html.

Jessop, Bob. 2007. Knowledge as a Fictitious Commodity: Insights and Limits of a Polanyian Perspective. In Reading Karl Polanyi for the Twenty-First Century. Market Economy as a Political Project, Hrsg. Ayse Bugra und Kaan Agartan, 115-133. New York: Palgrave Macmillan.

Kahin, Brian. 2003. What's Wrong with the Development of Intellectual Property Policy? In Die Zukunft der globalen Güter in der Wissensgesellschaft, Hrsg. Heinrich-BöllStiftung, 14-20. Berlin: Heinrich-Böll-Stiftung. 
Kaiser, Joachim W., und Alfred Schillert. 2005. Die Aufgaben einer PVA im Netzwerk der Erfindungsakquise und Patentverwertung. In Verwertungsnetzwerke. Eine Perspektive für den Technologietransfer, Hrsg. Michael Asche et al., 89-96. Münster: Waxmann.

Kamiyama, Shigeki, Jerry Sheehan, und Catalina Martinez. 2006. Valuation and Exploitation of Intellectual Property. STI Working Paper 2006/5. Paris: OECD.

Kenney, Martin. 2008. Reconsidering the Bayh-Dole Act and the Current University Invention Ownership Model. BRIE Working Paper 182. Berkeley: The Berkeley Roundtable on the International Economy.

Khan, B. Zorina. 2002. Intellectual Property and Economic Development: Lessons from American and European History. Study Paper 1a. Report to the IPR Commission. Cambridge, MA: National Bureau of Economic Research.

Khan, B. Zorina, und Kenneth L. Sokoloff. 2001. History Lessons. The Early Development of Intellectual Property Institutions in the United States. Journal of Economic Perspectives 15: 233-246.

Kienbaum 2006. Abschlussbericht. Weiterentwicklung von Kriterien sowie Datenerhebung auf der Basis der Kriterien und Datenauswertung bezüglich der Kompetenz und Leistungsfähigkeit der Patent- und Verwertungsagenturen. Düsseldorf: Kienbaum Management Consultants.

Kitch, Edmund W. 1980. The Law and Economics of Rights in Valuable Information. The Journal of Legal Studies 9: 683-723.

Kneller, Robert. 1999. Intellectual Property Rights and University-Industry Technology Transfer in Japan. In Industrializing knowledge. University-industry linkages in Japan and the United States, Hrsg. Lewis M. Branscomb, Fumio Kodama und Richard Florida, 307-347. Cambridge, MA: MIT Press.

Lamoreaux, Naomi R., und Kenneth L. Sokoloff. 2001. Market Trade in Patents and the Rise of a Class of Specialized Inventors in the 19th-Century United States. The American Economic Review 91: 39-44.

Lamoreaux, Naomi R., und Kenneth L. Sokoloff. 2007. The Market for Technology and the Organization of Invention in U.S. History. In Entrepreneurship, Innovation, and the Growth Mechanism of the Free-Enterprise Economies, Hrsg. Eytan Sheshinski, Robert J. Strom und William J. Baumol, 213-243. Princeton: Princeton University Press.

Landes, William M., und Richard A. Posner. 2003. The Economic Structure of Intellectual Property Law. Cambridge, MA: Harvard University Press.

Lemley, Mark A. 2005. Property, Intellectual Property, and Free Riding. Texas Law Review 83: 1031-1075.

Lerner, Josh. 2002. 150 Years of Patent Protection. The American Economic Review 92: 221-225.

Levin, Richard C., et al. 1987. Appropriating the Returns from Industrial Research and Development. Brookings Papers on Economic Activity 1987: 783-831.

Lichtenthaler, Ulrich. 2005. External commercialization of knowledge. Review and research agenda. International Journal of Management Reviews 7: 231-255.

Lichtenthaler, Ulrich, und Holger Ernst. 2008a. Intermediary Services in the Markets for Technology. Organizational Antecedents and Performance Consequences. Organization Studies 29: 1003-1035. 
Lichtenthaler, Ulrich, und Holger Ernst. 2008b. Innovation Intermediaries: Why Internet Marketplaces for Technology Have Not Yet Met the Expectations. Creativity and Innovation Management 17: 14-25.

Lipfert, Stephan, und Juliane Ostler. 2007. Fonds und Auktionen: Neue Formen der Patentverwertung. In Gewerbliche Schutzrechte im Innovationsprozess, Hrsg. Thomas Tiefel, 85-106. Wiesbaden: Deutscher Universitätsverlag.

Lyotard, Jean-François. 1984. The Postmodern Condition. Manchester: Manchester University Press.

Machlup, Fritz. 1984. The Economics of Information and Human Capital. Princeton: Princeton University Press.

Machlup, Fritz, und Edith Penrose. 1950. The Patent Controversy in the Nineteenth Century. Journal of Economic History 10: 1-29.

MacLeod, Christine. 1988. Inventing the Industrial Revolution. Cambridge: Cambridge University Press.

Malackowski, James E. 2006. The Intellectual Property Marketplace: Past, Present and Future. John Marshall Review of Intellectual Property Law 5: 605-616.

May, Christopher. 2000. A Global Political Economy of Intellectual Property Rights. The new enclosures? London: Routledge. (Siehe auch zweite geänderte Auflage 2010.)

May, Christopher. 2007. The hypocrisy of forgetfulness: The contemporary significance of early innovations in intellectual property. Review of International Political Economy 14: 1-25.

May, Christopher, und Susan K. Sell. 2006. Intellectual Property Rights. A Critical History. Boulder, CO: Lynne Rienner.

McCalman, Phillip. 2001. Reaping what you sow. An empirical analysis of international patent harmonization. Journal of International Economics 55: 161-186.

Meier, Henk Erik. 2005. Wissen als geistiges Eigentum? Die Erfindung der Public Domain. Leviathan 33: 492-521.

Merkel, Angela. 2007. Patente sind Zukunft. Rede auf dem European Patent Forum 2007 des Europäischen Patentamts. Berlin: Regierung online. http://www.bundesregierung.de/Content/DE/Archiv16/Rede/2007/04/2007-04-18-merkel-patentamt.html.

Möllering, Guido. 2009. Market Constitution Analysis. A New Framework Applied to Solar Power Technology Markets. MPIfG Working Paper 09/7. Cologne: Max Planck Institute for the Study of Societies.

Möllering, Guido. 2010. Collective Market-Making Efforts at an Engineering Conference. MPIfG Discussion Paper 10/2. Cologne: Max Planck Institute for the Study of Societies.

Mokyr, Joel. 2002. The Gifts of Athena. Historical Origins of the Knowledge Economy. Princeton: Princeton University Press.

Monk, Ashby H. B. 2009. The emerging market for intellectual property. Drivers, restrainers, and implications. Journal of Economic Geography 9: 469-491.

Mowery, David C., et al. 2001. The growth of patenting and licensing by U.S. universities. An assessment of the effects of the Bayh-Dole act of 1980. Research Policy 30: 99-119.

Mowery, David C., et al. 2004. Ivory Tower and Industrial Innovation. University-Industry Technology Transfer before and after the Bayh-Dole Act in the United States. Stanford: Stanford University Press. 
Nelson, Richard R. 2004. The market economy, and the scientific commons. Research Policy 33: 455-471.

Nicholas, Tom. 2003. Why Schumpeter was Right. Innovation, Market Power, and Creative Destruction in 1920s America. Journal of Economic History 63: 1023-1058.

Nonaka, I., und H. Takeuchi. 1995. The Knowledge-Creating Company: How Japanese Companies Create the Dynamics of Innovation. New York: Oxford University Press.

NRC, National Research Council. 2004. A Patent System for the 21st Century. Washington, D.C.: The National Academies Press.

Nuss, Sabine. 2006. Copyright \& Copyriot. Aneignungskonflikte um geistiges Eigentum im informationellen Kapitalismus. Münster: Westfälisches Dampfboot.

Orsi, Fabienne, und Benjamin Coriat. 2006. The New Role and Status of Intellectual Property Rights in Contemporary Capitalism. Competition or Change 10: 162-179.

Ostrom, Elinor. 1990. Governing the Commons. The Evolution of Institutions for Collective Action. Cambridge: Cambridge University Press.

Park, Walter G. 2008. International patent protection: 1960-2005. Research Policy 37: 761-766.

Park, Walter G., und D. C. Lippoldt. 2004. International Licensing and the Strengthening of Intellectual Property Rights in Developing Countries. OECD Trade Policy Working Papers 10. Paris: OECD.

PatVal-EU Project, Coordinator: Alfonso Gambardella. 2005. Study on Evaluating the Knowledge Economy. What are Patents Actually Worth? The Value of Patents for Today's Economy and Society. Final Report for Lot 1. Pisa: Laboratory of Economics and Management (LEM), Sant' Anna School of Advanced Studies.

Perez Pugatch, Meir. 2004. The International Political Economy of Intellectual Property Rights. Cheltenham: Edward Elgar.

Polanyi, Karl. 1957. The Great Transformation. The political and economic origins of our time. Boston: Beacon Press.

Polanyi, Michael. 1983. The tacit dimension. Gloucester, MA: Peter Smith.

PricewaterhouseCoopers. 2008. One Valuation fits all? Wie Europas innovativste Unternebmen Technologien und Patente bewerten. o. O.: PricewaterhouseCoopers.

Razgaitis, Richard. 2003. Valuation and Pricing of Technology-Based Intellectual Property. Chichester: John Wiley.

Reiffenstein, Tim. 2009. Specialization, Centralization, and the Distribution of Patent Intermediaries in the USA and Japan. Regional Studies 43: 571-588.

Reitzig, Markus. 2004. The Private Values of 'Thickets' and 'Fences': Towards an Updated Picture of the Use of Patents Across Industries. Economics of Innovation and New Technology 13: 457-476.

Rivette, Kevin G., und David Kline. 2000. Rembrandts in the Attic. Unlocking the Hidden Value of Patents. Boston, MA: Harvard Business School Press.

Scherer, Frederic M.. 2007. The Political Economy of Patent Policy Reform in the United States. Faculty Research Working Paper Series RWP07-042. Cambridge, MA: John F. Kennedy School of Government - Harvard University.

Schiff, Eric. 1971. Industrialization without National Patents: the Netherlands, 18691912; Switzerland, 1850-1907. Princeton: Princeton University Press.

Schneider, Ingrid. 2003. Funktionsgebundener Stoffschutz auf DNA-Sequenzen? Policyanalytische und wissenschaftstheoretische Anmerkungen zu einer zentralen Kontro- 
verse in der Biopatentierung.In Patente am Leben? Ethische, rechtliche und politische Aspekte der Biopatentierung, Hrsg. Christoph Baumgartner und Dietmar Mieth, 179211. Paderborn: mentis.

Schumpeter, Joseph A. [1943] 1996. Capitalism, Socialism \& Democracy. Abingdon: Routledge.

Sebald, Gerd. 2008. Offene Wissensökonomie. Analysen zur Wissenssoziologie der Freel Open Source-Softwareentwicklung. Wiesbaden: VS Verlag für Sozialwissenschaften.

Seckelmann, Margrit. 2006. Der „Dienst am schöpferischen Ingenium der Nation“. Die Entwicklung des Patentrechts im Nationalsozialismus. In Wirtschaftssteuerung durch Recht im Nationalsozialismus Hrsg. Johannes Bähr und Ralf Banken, 237-279. Frankfurt a. M.: Klostermann.

Sell, Susan K. 1999. Multinational Corporations as Agents of Change. The Globalization of Intellectual Property Rights. In Private Authority and International Affairs, Hrsg. A. Claire Cutler, Virginia Haufler und Tony Porter, 169-197. New York: State University of New York Press.

Sell, Susan K. 2003. Private Power, Public Law. The Globalization of Intellectual Property Rights. Cambridge: Cambridge University Press.

Serrano, Carlos J. 2006. The Market for Intellecual Property. Evidence from the Transfer of Patents. Working Paper. Toronto: University of Toronto.

Siegrist, Hannes. 2006. Geschichte des geistigen Eigentums und der Urheberrechte. Kulturelle Handlungsrechte in der Moderne. In Wissen und Eigentum. Geschichte, Recht und Ökonomie stoffloser Güter, Hrsg. Jeanette Hofmann, 64-80. Bonn: Bundeszentrale für politische Bildung.

Stehr, Nico. 1994. Knowledge Societies. London: SAGE.

Stehr, Nico. 2002. Knowledge and Economic Conduct. Toronto: University of Toronto Press.

Tietze, Frank, und Cornelius Herstatt. 2009. Intermediaries and Innovation. Why they emerge and how they facilitate IP Transactions on the Markets for Technology. Working Paper 59. Hamburg: Hamburg University of Technology.

Troy, Irene, und Raymund Werle. 2008. Uncertainty and the market for patents. MPIfG Working Paper 08/2. Cologne: Max Planck Institute for the Study of Societies.

UNESCO. 2005. Towards Knowledge Societies. Paris: UNESCO.

US Government Accounting Office. 1998. Technology Transfer. Administration of the Bayh-Dole Act by Research Universities. Report to Congressional Committees. May 7, 1998.

US Small Business Administration. 2009. Programs and Services. http://www.sba.gov/ services/index.html.

Van de Ven, Andrew H., et al. 1999. The Innovation Journey. New York: Oxford University Press.

Von Hippel, Eric. 1994. "Sticky Information" and the Locus of Problem Solving: Implications for Innovation. Management Science 4: 429-439.

Weißbach, Hans-Jürgen. 2010. Die Patentierung von Wissensgütern. Effektiver Wissensschutz oder Trivialisierung der Wissensproduktion. In Wissensökonomie und Innovation, Hrsg. Manfred Moldaschl und Nico Stehr, 331-370. Marburg: Metropolis. 
Werle, Raymund. 2005. Der Schutz des geistigen Eigentums in der Medien- und Softwareindustrie im Interessen- und Wertkonflikt. In Ethik der Informationsgesellschaft, Hrsg. Martin Woesler, 73-104. Bochum: Europäischer Universitätsverlag.

West, Joel. 2006. Does Appropriability Enable or Retard Open Innovation? In Open Innovation, Hrsg. Henry Chesbrough, Wim Vanhaverbeke und Joel West, 109-133. Oxford: Oxford University Press.

Williamson, Oliver E. 1979. Transaction-Cost Economics: The Governance of Contractual Relations. Journal of Law and Economics 22: 233-261.

Willke, Helmut. 2007. Wissensgesellschaft. Kollektive Intelligenz und die Konturen eines kognitiven Kapitalismus. In Kognitiver Kapitalismus. Soziologische Beiträge zur Theorie der Wissensökonomie, Hrsg. Hanno Pahl und Lars Meyer, 195-221. Marburg: Metropolis.

Ziedonis, Rosemarie Ham. 2008. On the Apparent Failure of Patents. A Response to Bessen and Meurer. Academy of Management Perspectives 22: 21-29.

Zucker, Lynne G., Michael R. Darby, und Marilynn B. Brewer. 1998. Intellectual Human Capital and the Birth of U.S. Biotechnology Enterprises. The American Economic Review 88: 290-306. 